\title{
BIENESTAR SUBJETIVO DE MIGRANTES: LA AUSENCIA DE LA DIMENSIÓN DE GÉNERO
}

\author{
MIGRANTS' SUBJECTIVE WELL-BEING: \\ THE ABSENCE OF THE GENDER DIMENSION
}

ROBERTA PANZERI*

\begin{abstract}
RESUMEN
El propósito de este artículo es comprender el estado del arte de los estudios que se han ocupado de la influencia que la experiencia migratoria tiene en el desarrollo de las capacidades y la satisfacción con la vida. En particular, quiere averiguar si están cubiertos todos los aspectos relevantes relacionados con el bienestar subjetivo de los migrantes. Se lleva a cabo una revisión bibliográfica sistemática de los estudios sobre este tema y se analizan de forma detallada las variables recogidas. Emerge la preponderancia de estudios cuantitativos y la falta de una perspectiva de género. Los resultados hacen evidente la necesidad de una investigación con un foco específico sobre el bienestar subjetivo de las migrantes.
\end{abstract}

PALABRAS CLAVE: migración internacional, bienestar subjetivo, género.

\begin{abstract}
The aim of this article is to understand the state of the art of investigation on the influence that migratory experience has on the capabilities' development and satisfaction with life, in terms of perceived personal fulfillment and achievement of individual goals. In particular, it wants to find out whether all relevant aspects related to $\mathrm{mi}$ grants' subjective well-being are covered. A systematic bibliographic review of the studies on this subject is carried out and the collected variables are analysed in detail. The preponderance of quantitative studies and the lack of a gender perspective emerge, since the gender variable, when present, is used only as a control variable. Results show the need for research with a specific focus on the subjective well-being of migrant women.
\end{abstract}

KEYWORDS: international migration, subjective well-being, gender perspective.

\footnotetext{
* Máster en Derechos Humanos, Interculturalidad y Desarrollo. Facultad de Ciencias Políticas y Sociología. Universidad de Granada, España. C/ Rodrigo de Triana, 65 - 41010. Sevilla, España.

E-mail address: robertapanzeri@correo.ugr.es
} 


\section{INTRODUCCIÓN}

La migración humana ha sido un fenómeno omnipresente desde los comienzos de la humanidad. Hoy, como siempre ha ocurrido, la migración es el resultado de numerosos factores, el principal de los cuales es la búsqueda de oportunidades para mejorar las condiciones de vida, por ejemplo, para vivir en un entorno más favorable y próspero en recursos, agradable o seguro, o reunirse con miembros del mismo grupo social.

En el último medio siglo, la migración internacional ha aumentado en todo el mundo; el número de personas que viven fuera de sus países de origen aumentó de 75 millones en 1975 (representando el 2,2 \% de la población mundial) a 244 millones en 2015 (lo que representa el 3,3\% de la población mundial), y se estima que se llegará a más de 230 millones en la década de 2050 (OIM, 2010).

$\mathrm{Si}$ bien un considerable porcentaje de migrantes no se desplaza por voluntad propia, sino que se ve obligado a escapar de sus hogares, cabe pensar que una gran variedad de personas perciben la posibilidad de mudar el lugar donde vivir, como una valiosa oportunidad para mejorar su vida. En ambos casos, la migración a otro país es una de las decisiones más impactantes en la vida, ya que rompe los patrones sistemáticos en la esfera laboral, en la vida social y en el ambiente externo de una persona.
Para quienes afrontan la migración, por lo tanto, las cuestiones de mayor trascendencia son si serán más felices tras emigrar y si su vida (o la de sus familias) será mejor que antes.

En las últimas décadas se ha señalado que el bienestar es algo más complejo que el simple crecimiento económico y se ha hecho imprescindible dar mayor relevancia al bienestar subjetivo.

El interés por la felicidad y el bienestar como resultado de la migración es muy reciente, y su incremento repentino se hace evidente en la desproporcionada propagación de los artículos publicados sobre el tema en los últimos diez años, sobre todo en revistas de sociología y psicología (Hendriks, 2015). Estudiar la felicidad posmigratoria siempre ha sido bastante complejo, ya que el argumento se ha mantenido excepcionalmente multidisciplinario (se ha abordado desde la psicología, la sociología y la economía), disperso y fragmentado, y a lo largo del tiempo ha producido una multiplicidad de vocablos para denominar el tema.

El objetivo de este trabajo es proporcionar una fotografía del estado actual de la investigación sobre la satisfacción posmigratoria, y poner de relieve las lagunas o los intersticios que queden vacíos e inexplorados. Esto nos llevará a evidenciar la necesidad de una perspectiva que explore el bienestar subjetivo de los individuos en un sentido amplio y multidi- 
mensional, para evitar aplanar o simplificar las situaciones personales dentro de generalizaciones o abstracciones.

En la siguiente sección se exponen las principales construcciones teóricas que sustentan la investigación. La tercera, sección presenta la metodología utilizada para la exploración bibliográfica. La cuarta sección indica y discute los hallazgos de la revisión. En la última, se presentan las conclusiones y se discuten las exigencias para la investigación futura.

\section{MARCO TEÓRICO}

\subsection{Bienestar Subjetivo}

En las últimas décadas, se hizo manifiesta la necesidad de incluir instrumentos alternativos al PIB para medir el progreso social, lo que ha extendido la idea de que el bienestar de las personas va más allá del bienestar económico. En la literatura se encuentra una gran variedad de términos para referirse al concepto de bienestar: Calidad de vida (Cummins, 2010), desarrollo humano (M. C. Nussbaum, 2011; Robeyns, 2005; Sen, 1985), bienestar subjetivo (E. Diener et al., 1999), Felicidad (Bartram, 2013; Hendriks, 2015), pero también Life Satisfaction (Amit, 2010; Baykara-Krumme y Platt, 2016; Goodwin, Cook, y Yung, 2001; Nesterko, Braehler, Grande, y Glaesmer, 2013; Safi, 2010; Verkuyten, 2008; Voicu y Vasile, 2014; Vroome y Hooghe, 2014) y Utility (Kahneman, 2012).
Asimismo, hay varias teorías o relatos competidores que intentan especificar la naturaleza profunda del concepto de bienestar. Raibley (2012) por ejemplo enumera las diversas versiones del hedonismo (Feldman, 2004), la satisfacción del deseo (Murphy, 1999), la teoría de la lista objetiva (Arneson, 1999), el alcance del logro (Keller, 2009; Scanlon, 1998), el desarrollismo (Kraut, 1979), la teoría de la satisfacción (Sumner, 1996), la teoría de la felicidad sostenible (Lyubomirsky, Sheldon, y Schkade, 2005), la teoría de la auto-realización (Haybron, 2008) y el enfoque de las capacidades (Nussbaum, 2001; Nussbaum y Sen, 1993; Sen, 1999). También se han ocupado de bienestar, la teoría de desarrollo a escala humana Max-Neef et al. (1994), y las experiencias flow descritas por Csíkszentmihályi (2008).

Vale la pena resumir los puntos básicos de las principales teorías utilizadas en el estudio del bienestar.

Una de ella es la teoría de la lista objetiva (Arneson, 1999), que introduce el concepto de "valor prudencial", para subrayar que una persona debería buscar su propio bien de manera eficiente, seleccionando los mejores medios disponibles para su bien, ya que puede haber algún conflicto entre lo que es bueno para una persona y lo que es bueno para otras, y lo que exige la moralidad. 
La teoría del alcance del logro (Keller, 2004) añade que el bienestar de un individuo se fortalece tan pronto como se alcanzan sus objetivos, incluso cuando estos son insanos, autodestructivos, irracionales o inmorales.

Para la teoría de la satisfacción del deseo (Murphy, 1999), el bienestar de un agente está constituido por la obtención de los estados de cosas deseados por ese agente, pero el bienestar no residiría en la satisfacción de los deseos reales del agente, sino en la satisfacción de los deseos del agente en una "situación hipotética de deseo", es decir, un entorno óptimo para el deseo.

La Teoría de la felicidad sostenible (Lyubomirsky, Sheldon, y Schkade, 2005) propone que el nivel de felicidad crónica de una persona se rige por tres factores principales: un "punto de referencia" genéticamente determinado; factores circunstanciales o contextuales relevantes para la felicidad; actividades y prácticas intencionales. El "punto de referencia" refleja rasgos de personalidad intrapersonal, temperamental y afectiva relativamente inmutables que cambian poco a lo largo de la vida.

Las 'circunstancias' se refieren a los factores incidentales pero relativamente estables de la vida de un individuo (como el estado civil, el estado ocupacional, la seguridad laboral, los ingresos, la salud y las afiliaciones religiosas). Las "actividades intencionales" se refieren a una categoría amplia que incluye las actividades voluntarias y los esfuerzos que las personas realizan en su vida cotidiana. Los autores distinguen tres tipos de actividades intencionales o actos de esfuerzo: cognitivos (como evitar la comparación social, desarrollar estrategias de afrontamiento), conductuales (como alimentar el apoyo social), y volitivos (como el compromiso con los objetivos).

Según este modelo, el "punto de referencia" $\mathrm{y}$ las actividades intencionales representarían la mayor parte de la varianza en el bienestar (90 \%), mientras que las circunstancias solo contribuirían con el $10 \%$. Los cambios en las circunstancias tendrían un potencial limitado para producir cambios sostenibles en el bienestar, ya que las personas tendrían a adaptarse a circunstancias constantes.

El enfoque de las capacidades, conocido como capability approach, que, en sus distintas versiones (Nussbaum, 2011; Robeyns, 2005; Sen, 1985), reúne diferentes niveles de análisis de la experiencia subjetiva en una visión integrada y sensible, convirtiendose en una herramienta multidimensional para el estudio del bienestar, no solo a partir del aspecto económico. El enfoque de las capacidades pone de relieve que el objetivo del desarrollo y progreso social debe ser el de mejorar las libertades individuales y pone el énfasis en las capacidades como 
unidades básicas para el desarrollo humano. El aumento de dichas capacidades expande la libertad para vivir el tipo de vida que cada uno considera valedera, ya que amplía las posibilidades de alcanzar los objetivos que cada cual considera relevantes en su vida (Sen, 1985). Tomando como referencia las conceptualizaciones formuladas en el enfoque de las capacidades, Allardt (1993) ha operacionalizado un modelo para estudiar -a través de tres dimensiones: tener, amar, ser- el nivel de bienestar subjetivo dentro de una sociedad.

Otro importante modelo para el estudio del bienestar subjetivo (Diener y Suh, 1997; Kahneman y Krueger, 2006) es el que se ha desarrollado desde la tradición utilitaria de la psicología. Este modelo estudia el bienestar subjetivo a través de las siguientes dimensiones:

- La evaluación cognitiva de la satisfacción global de una persona con su vida;

- El bienestar experimentado, que se refiere a los estados de ánimo, los sentimientos y las emociones experimentadas, tanto positivas como negativas.

- El bienestar eudaimónico, es decir, el funcionamiento psicológico y floreciente (por ejemplo, los sentimientos de autoestima y competencia) y el sentido que una persona proporciona a su vida (Huppert et al., 2009; Raibley, 2012).

Para evaluar el bienestar subjetivo, la ma- yoría de las encuestas actualmente existentes se han elaborado a partir de estas últimas teorías.

El OECD ha elaborado una investigación internacional titulada "How's Life?: Measuring Well-being", que repite cada dos años desde el 2011, en la que pretende calcular el bienestar de la población de los 35 países miembros (OECD, 2015). Establece sus fundamentos teóricos integrando las reflexiones de Fitoussi et al., (2009), el concepto de bienestar subjetivo como meta para la existencia humana de la tradición utilitaria de la psicología (Diener \& Suh, 1997), el enfoque de las capacidades (Sen, 1985, 1987). De esta manera, la OECD (2011) considera el bienestar de las personas como el resultado de dos grandes dimensiones: las condiciones materiales de vida (o "bienestar económico”) y la calidad de vida (el conjunto de atributos no monetarios de los individuos, que moldea sus potencialidades y oportunidades de vida, y tiene valor intrínseco bajo diferentes culturas y contextos). El bienestar subjetivo se encuentra, entre otros indicadores, bajo la dimensión de calidad de vida y se considera complejo de medir, ya que se refiere a la percepción de las personas.

A su vez, el European Research Infrastructure Consortium ha confeccionado una encuesta científica internacional que se realiza cada dos años desde 2001, a través de la cual se proporcionan indicadores sobre el progreso de los diferentes 
países, basados en las percepciones de los ciudadanos acerca de los aspectos clave de sus sociedades (ESS, s. f.). Las dimensiones propuestas son: bienestar evaluativo, bienestar emocional, funcionamiento, vitalidad, bienestar comunitario, relaciones de apoyo.

Otra importante referencia europea es la European Quality of Life Survey (Eurofound, 2003) que explora varios aspectos como: empleo, ingresos, educación, alojamiento, familia, salud, equilibrio trabajo-vida, felicidad, satisfacción de vida y sentido de pertenencia, donde los últimos tres aspectos son los que definen el bienestar subjetivo.

Para medir el bienestar subjetivo también se han utilizado algunas preguntas presentes en la World Values Survey y en la Gallup World Poll (Gallup, s. f.).

\subsection{Bienestar subjetivo de las personas migrantes}

La mayor parte de la literatura científica sugiere que la migración es uno de los eventos estresantes más significativos de la vida, ya que los migrantes se enfrentan a múltiples factores angustiosos, como la pérdida de redes sociales, familiares y de apoyo, la discriminación y el subempleo, además de la barrera lingüística y las diferentes normas culturales. Por esta razón el efecto de la migración sobre el bienestar psicológico y la aparición de angustia, ansiedad y depresión ha sido ampliamente estudiado. Sin embargo, se ha prestado poca atención a los aspectos más positivos de la migración y, en particular, a su efecto sobre el bienestar. La evidencia apoya la noción de que los inmigrantes pueden ser saludables, resistentes y capaces de responder positivamente a los peligros potenciales para la salud de la migración (Bak-Klimek et al., 2015).

En este sentido, los migrantes son un grupo autoseleccionado que tiene características peculiares propias.

Asimismo, la felicidad de los migrantes depende también de factores que no afectan a los no migrantes, como la aculturación, la doble pertenencia, las habilidades sociales necesarias para reconstruir una red social y económica en un entorno nuevo y las condiciones de su país de origen. Por último, la felicidad de los migrantes puede depender en mayor medida de las razones específicas que motivaron su traslado (por ejemplo, la relación familiar y de pareja para la reunificación familiar).

Por todas estas razones, los hallazgos de la literatura general sobre la felicidad y el bienestar subjetivo dan pistas muy útiles, pero no son suficientes para estudiar el bienestar de los migrantes, ya que las funciones de felicidad de este colectivo pueden diferir de las de la población en general y no sabemos hasta qué punto las teorías generales del bienestar se ven corroboradas por la investigación llevada a cabo con inmigrantes (Hendriks y Bartram, 2018). 
Por lo tanto, para tener un cuadro más completo del estado actual de la investigación, a las anteriores teorías hay que añadir algunas más específicas que nos pueden ayudar para comprender la situación de los migrantes.

Por ejemplo, la teoría de las cuatro "estrategias de aculturación” (asimilación, separación, marginación e integración) de Berry (1997), establece que la calidad de la aculturación de un migrante en el entorno cultural de acogida depende de su capacidad para hacer frente de manera adecuada y eficaz a las tensiones asociadas con la adaptación cultural. En esta misma estría se pone el paradigma de la asimilación en "línea recta", que afirma que la felicidad de los migrantes mejora al aumentar la duración de su estancia, ya que asume que los migrantes se vuelven cada vez más parecidos a los nativos a medida que pasan los años (Richardson, 1974). En este sentido, la asimilación podría considerarse como el proceso de reducción del dolor psicológico inherente a la experiencia de la migración.

La teoría de la habitabilidad de Veenhoven (2000) propone una clasificación basada en dos bi-particiones: entre las "oportunidades" y los "resultados" en la vida, y entre las cualidades "interiores" y "exteriores". Las combinaciones de estas dos dicotomías dan lugar a cuatro cualidades de vida: 1) la habitabilidad del medioambiente, 2) la habitabilidad del individuo,
3) la utilidad externa de la vida, y 4) la apreciación interna de la vida.

La habitabilidad es el grado en que un entorno vital encaja en el repertorio adaptativo de una especie. Aplicado a la sociedad humana, denota el encaje de los arreglos institucionales con las necesidades y capacidades humanas. La teoría de la habitabilidad explica las diferencias observadas en la felicidad en términos de ajuste entre necesidad y medioambiente.

Otro enfoque teórico que se ha tomado en consideración para el estudio del bienestar de los migrantes es el conocido como la paradoja de Easterlin (1974) que consiste en que cuando la renta (y por lo tanto el bienestar económico) crece, la felicidad humana aumenta hasta cierto punto, pero luego comienza a disminuir, siguiendo una curva parabólica con concavidad hacia abajo.

Otra importante teoría es la de la comparación social (Festinger, 1954), que argumenta que los individuos evalúan sus propias opiniones y capacidades mediante la comparación con otros con el fin de reducir la incertidumbre en esos ámbitos, y aprender a definirse a uno mismo.

Esta teoría asume que la felicidad resulta de comparaciones entre nociones de cómo debería ser la vida y cómo es en realidad, y supone que cuanto mayor sea la brecha entre lo que uno quiere y lo que uno tiene, menos feliz es. En esta teoría, los "de- 
seos" difieren de las "necesidades". En primer lugar, los deseos son sostenidos conscientemente, mientras que uno puede ser inconsciente de lo que necesita. Asimismo, los deseos son construcciones sociales y, como tales, pueden variar de una cultura a otra, mientras que las necesidades son universales. Finalmente, los deseos tienden a ser interminables, mientras que las necesidades pueden ser satisfechas. Este último punto implicaría que una gran felicidad no es posible, ya que si siempre queremos más de lo que tenemos, nunca seremos más felices.

Es por esta razón que la búsqueda de la felicidad ha sido también representada como la "rueda de ardilla hedónica".

\subsection{Bienestar subjetivo de las mujeres migrantes}

Las transformaciones del mercado de trabajo a escala global, y la creciente demanda de trabajadoras han llevado a una progresiva feminización de los movimientos migratorios. El porcentaje de mujeres entre los migrantes internacionales en Europa, Norteamérica y Oceanía ha pasado del $47,9 \%$ en 1960 a 50,9 \% en 2000. Esto ha desencadenado un interés creciente por parte de la literatura científica sobre la migración femenina, es decir que se han multiplicado los estudios sobre género y migración.

Sin embargo, no se encuentra una literatura que se ocupe de forma específica sobre el bienestar subjetivo de las mujeres mi- grantes. De ahí la pregunta: ¿̇los factores considerados importantes para el bienestar de las mujeres serán los mismos factores que influyen en el bienestar de los hombres? Por ejemplo, dlas razones que empujan a la migración son las mismas entre hombres y mujeres? ¿Y la percepción de la diferencia en sus circunstancias de vida anteriores o posteriores a la migración? ¿O los aspectos que califican como importantes para considerarse satisfechos? ¿Tienen el mismo peso los factores relacionales y familiares? Todos estos factores nos proporcionan importantes informaciones para reflexionar sobre cuáles son los elementos que condicionan la calidad de vida de una mujer, y la hacen valedera.

En el Informe sobre la felicidad en el mundo de 2015 (Fortin et al., 2015), producido por la Red de Soluciones para el Desarrollo Sostenible de las Naciones Unidas, entre las seis variables utilizadas para explicar las diferencias internacionales en las evaluaciones de la vida -apoyo social, libertad, generosidad, corrupción, problemas de salud e ingresos del hogar- se pueden notar algunas diferencias de género. Por ejemplo, el apoyo social (tener a alguien con quien contar) tiene una tendencia a la baja con la edad para ambos sexos, pero con una recuperación posterior que es mayor para las mujeres que para los hombres. Los hombres encuestados informan que sus ingresos familiares son más elevados que 
los de las mujeres en los grupos de edad hasta finales de la mediana edad.

Otro resultado interesante es que los sentimientos de tristeza son siempre bastante más comunes entre las mujeres que entre los hombres. La mayor frecuencia de tristeza expresada para las mujeres que para los hombres es un hallazgo estándar en la psicología experimental. Se atribuye de diversas maneras a las diferencias en la socialización, a las diferencias inherentes de género en la emocionalidad y a una mayor disposición de las mujeres a discutir los problemas emocionales.

En dos regiones -Europa Occidental y América Latina- el estrés reportado es siempre significativamente más frecuente para las mujeres que para los hombres. La preocupación por las mujeres es generalmente más frecuente que la ira, y aumenta en todos los grupos de edad, del $25 \%$ entre las mujeres más jóvenes al $37 \%$ entre los grupos de edad más avanzada. Por último, En Europa Occidental y América Latina se observan tasas de depresión significativamente más altas y crecientes entre las mujeres.

Asimismo, el enfoque de las capacidades hace hincapié en la importancia de la libertad de elección, la heterogeneidad individual y la naturaleza multidimensional del bienestar. Las variaciones interpersonales, debidas tanto a factores personales como a factores socio-ambientales, pueden dar lugar a grandes diferencias en la conversión de las oportunidades en funcionamientos. Robeyns (2005) nos recuerda que se pueden distinguir tres tipos de factores de conversión que son fundamentales a la hora de convertir unos bienes en funcionamientos reales, es decir las características personales (la condición física, el sexo, la inteligencia), las características sociales (las políticas públicas, las normas sociales, las prácticas discriminatorias, los roles de género, las jerarquías sociales, las relaciones de poder) y las características ambientales (el clima, las infraestructuras, las instituciones, los bienes públicos). Para la evaluación de las desigualdades de género en las sociedades occidentales posindustrializadas, Robeyns (2003) incluye explícitamente el funcionamiento de la autonomía de tiempo, la distribución del tiempo, el tiempo libre y el estrés relacionado con el tiempo.

Por último, los estudios económicos que tienen en cuenta el papel de la familia como unidad de migración nos informan que hay dos tipos de migración: separada o conjunta. La migración separada se refiere a la migración de uno o más miembros del hogar, pero no de la unidad entera. La migración conjunta es la migración de toda la unidad familiar en su conjunto. A veces las ganancias individuales no son iguales para todos los miembros de la familia. En estos casos hay que tener en cuenta los roles y la desigualdad de género y el poder en la toma de decisiones 
incluso dentro de los hogares. Por ejemplo, a menudo las mujeres son "seleccionadas" para emigrar por sus familias, ya que se cree que saben sacrificarse en un grado mayor que los hombres por el bienestar.

\section{MÉTODO}

En este trabajo se presenta una exploración sistemática de la literatura en el tema de bienestar subjetivo de las personas migrantes.

Los documentos que relacionan la migración con la felicidad se publican en revistas de múltiples disciplinas, incluyendo las que se centran en la migración, el bienestar subjetivo, la economía, la psicología y la sociología. Se realizó una búsqueda sistemática de artículos en inglés utilizando la base de datos Web of Science, Scopus y Google Scholar, mediante palabras clave relacionadas con la migración y con el bienestar, en particular se usaron las siguientes palabras clave así combinadas: "International migration" y "Happiness", o "Life Satisfaction", o "Subjective well-being". Se analizaron los principales y más recientes artículos en temas de bienestar subjetivo de los migrantes, relacionados con el área geográfica europea. Esta búsqueda produjo catorce estudios. Consecuentemente se examinaron los artículos relevantes citados en los documentos ya incluidos, lo que llevó siete estudios adicionales, para un total de 21 artículos. El siguiente paso fue examinarlos y analizar las siguientes variables: el marco conceptual del estudio, el área geográfica de referencia (países incluidos en el estudio); el método de investigación utilizado; la tipología y las fuentes de los datos; la población de estudio; la utilización de la dimensión de género; los resultados relacionados a la dimensión de género en los migrantes; los parámetros utilizados para medir el Bienestar Subjetivo (BS); las preguntas de la encuesta para evaluar el SB (escala de puntuación) y por último los resultados principales del estudio.

\section{RESULTADOS}

En la pagina 51 presentamos la Tabla con el análisis de los 21 estudios empíricos seleccionados.

\section{DISCUSIÓN Y VALORACIÓN DE HALLAZGOS}

A pesar de que los 21 estudios examinados siguen enfoques heterogéneos en cuanto a preguntas de investigación, elección de la población a comparar, países cubiertos y fuentes de datos, hay algunas características comunes y recurrentes que emergen claramente. Más concretamente, podemos destacar al menos cuatro dimensiones.

(1) Fuentes de datos. El uso de encuestas internacionales, bien establecidas, con una larga historia (plurianual) y diferentes oleadas prevalece decisivamente. En par- 
ticular, ocho de los estudios examinados utilizan la encuesta ESS, mientras que cuatro utilizan la encuesta Gallup World Poll. Esas encuestas abarcan amplias muestras representativas de la población e incluyen un número de migrantes que, en términos absolutos, es lo suficientemente elevado como para proporcionar una masa crítica de observaciones y un cierto grado de flexibilidad para llevar a cabo eficazmente análisis cuantitativos. Cinco estudios utilizan encuestas diferentes, en su mayoría nacionales, mientras que solo uno de ellos utiliza una hecha ad hoc, y otro se basa en entrevistas presenciales.

(2) Medidas de bienestar subjetivo utilizadas. La mayoría de los estudios (20 de 21) utilizan la satisfacción con la vida $y / 0$ la felicidad general como medidas empíricas del bienestar subjetivo. En particular, 10 estudios se basan enteramente en la satisfacción con la vida y cuatro en la felicidad general; los restantes estudios utilizan más de un indicador. Cabe señalar que la encuesta ESS incluye preguntas específicas para evaluar tanto la satisfacción con la vida como la felicidad general, y todos los estudios que utilizan la felicidad como indicador de bienestar subjetivo se basan en esta encuesta. En cambio, la encuesta Gallup World Poll no incluye preguntas específicas sobre la felicidad. La mayoría de los estudios derivan su medida de bienestar subjetivo basándose en la respuesta a una sola pregunta planteada en las encuestas (13 de 21) y expresada en una escala discreta (la mayoría de las veces una escala de o a 10). Algunos estudios combinan respuestas a diferentes preguntas, o bien en un único indicador más sofisticado (multidimensional) o bien por separado (6 estudios de 21).

(3) Equilibrio entre los estudios cuantitativos y cualitativos. Todos los estudios empíricos examinados son de naturaleza cuantitativa. Solo hay un documento que se declara cualitativo (D'Isanto et al., 2016), pero es en realidad cuantitativo, ya que traduce las respuestas proporcionadas durante entrevistas en indicadores cuantitativos y luego realiza un análisis estadístico utilizando técnicas de comparación de puntajes de propensión para comparar los grupos de población.

(4) Marcos teóricos (principales) utilizados. La literatura examinada presenta un patrón muy heterogéneo. Sin embargo, algunos enfoques parecen estar más presentes que otros; en particular, tres estudios hacen referencia al enfoque de Diener, otros tres mencionan la paradoja de Easterlin como marco de referencia y otros cuatro se basan sobre los estudios de Veenhoven.

En resumen, la literatura empírica examinada en este trabajo puede caracterizarse como: esencialmente de naturaleza cuantitativa, basada en encuestas internacionales y en respuestas a un número 
muy reducido de preguntas para cuantificar el bienestar subjetivo, ya sea en términos de satisfacción de la vida o de felicidad general.

\subsection{Hallazgos generales}

En términos de resultados generales en relación con el bienestar subjetivo de los migrantes, destacan los siguientes elementos. En primer lugar, la mayoría de los documentos que hacen una comparación entre migrantes y nativos encuentran que los migrantes tienden a reportar un nivel más bajo de satisfacción y/o felicidad que los nativos (8 de 21); mientras que Voicu y Vasile (2014) sugieren que el proceso de ajuste del bienestar subjetivo en los países de acogida es dinámico y puede converger al nivel de los nativos en el largo plazo. Dos estudios centrados en el cambio en el bienestar subjetivo de los migrantes (D'Isanto et al., 2016; Nikolova y Graham, 2015) encuentran que la migración aumenta el bienestar subjetivo. Dos estudios (Hendriks, 2015; OIM, 2013) destacan que el cambio (aumento) experimentado en el bienestar subjetivo después de la migración depende en gran medida de la corriente o ruta específica de migración y, por lo tanto, no puede asumirse como un hallazgo general; en particular, es probable que los migrantes Norte-Norte alcancen un nivel similar de satisfacción con la vida que los nativos, mientras que todos los demás migrantes tienen menos probabilidades de alcanzar y exhibir un nivel de bienestar subjetivo similar al de los nativos. Otros estudios destacan la importancia de diferentes factores para determinar el bienestar subjetivo de los migrantes: condiciones sociales y económicas (Hendriks y Bartram, 2016; Olgiati et al., 2013; Vroome y Hooghe, 2014); diferentes niveles de satisfacción y felicidad en los países de origen antes de la migración, señalando la importancia de los fenómenos de autoselección (Bartram, 2013; Hajdu y Hajdu, 2016; Ivlevs, 2015); factores individuales como la edad de migración (Nesterko et al., 2013), o factores de disposición y cognitivo-comportamentales (Bak-Klimek et al., 2015).

\subsection{Hallazgos en materia de género}

Con respecto a la dimensión de género, en la revisión de los estudios empíricos destacan cinco elementos principales.

(1) En primer lugar, la información sobre el género está presente en todas las fuentes de datos utilizadas en los 21 estudios examinados. Se trata de un hecho importante, ya que permite en principio el uso de dicha información en el diseño y la implementación del análisis empírico y deja enteramente en manos del investigador la elección de si y cómo explotar dicha dimensión tanto en la formulación de las preguntas de investigación como en el contexto analítico.

(2) Ninguno de los estudios examinados ha sido diseñado explícitamente para 
abordar la relación entre el género y el bienestar subjetivo en general, y mucho menos el bienestar subjetivo de los migrantes. Aunque la dimensión de género entra menudo en el análisis (véase el siguiente punto), no se aborda de antemano en ninguno de los estudios, ni forma parte integrante de la pregunta principal de investigación. Sin embargo, algunos estudios dedican más atención que otros a este tema. Por ejemplo, Bak-Klimek et al. (2015) en su revisión de la literatura encuentra que en cinco de los 12 estudios cuantitativos examinados, el género se utiliza como predictor del bienestar subjetivo de los migrantes, aunque no parece ser un factor estadísticamente significativo. D'Isanto et al. (2016) también usan el género como predictor de la satisfacción con la vida y no encuentran resultados significativos.

(3) Cuando la dimensión de género está explícitamente presente en el análisis empírico (15 estudios), se utiliza principalmente como variable de control o variable de emparejamiento para identificar pares equivalentes de migrantes-no migrantes (13 estudios). En otras palabras, se utiliza la dimensión de género para eliminar los efectos "espurios" que pueden desdibujar la relación principal que es objeto del estudio. De esta manera, el género entra en el cuadro analítico solo como instrumento para obtener otros resultados y a menudo es ignorado en la discusión.
(4) Cuando se incluye como control en los modelos que explican la diferencia en el bienestar subjetivo entre migrantes y no migrantes, y cuando los resultados estan explícitamente reportados y/o comentados, la variable de género es estadísticamente significativa en seis casos, mientras no lo es en cuatro casos. En los seis casos positivos, ser mujer tiene invariablemente un efecto positivo, ya sea en la satisfacción con la vida o en la felicidad general, aunque el efecto es pequeño. Este resultado sugiere un posible efecto de género también entre los migrantes, que no se explora en los estudios examinados.

(5) Notablemente, los principales informes de las instituciones internacionales, OIM (2013) y OCDE (2017), no dedican una sección específica a la dimensión de género del bienestar subjetivo de los migrantes, a pesar de que tratan de cubrir exhaustivamente todas las facetas de la relación entre migración y bienestar.

\section{CONCLUSIONES}

El presente trabajo proporciona un mapa de los estudios actualmente presentes en la literatura e identifica algunas lagunas que podrían cubrirse en estudios posteriores.

Los estudios cubiertos en la presente revisión presentan una amplia gama de posibles enfoques, revelando la riqueza de este campo de investigación. 
Existe una clara brecha en la literatura con respecto al uso de estudios cualitativos. En particular, parece haber margen para utilizar enfoques más cualitativos a fin de mejorar la construcción de las medidas de bienestar subjetivo, que en los estudios examinados se basan principalmente en una sola respuesta a una pregunta de encuesta, parametrizada en una escala discreta. El empleo de una medida unidimensional del bienestar es una simplificación útil para el análisis cuantitativo, pero que sin duda dificulta el descubrimiento de dinámicas más profundas y matizadas que pueden ser cruciales para comprender los factores que realmente determinan el bienestar subjetivo.

Asimismo, se destaca la falta de un diseño empírico específico destinado a captar las diferencias de género, aunque haya algunas pruebas de que esta dimensión es importante. La escasa atención al papel del género puede deberse a la dificultad objetiva de combinar tres dimensiones analíticas: bienestar subjetivo, género y migración. Sin embargo, constituye un verdadero hueco en la producción científica relativa a las cuestiones conjuntas (interdisciplinarias) de género y migración al considerar los enfoques de bienestar. El artículo esclarece por lo tanto la necesidad de ampliar el abanico de preguntas que deben formularse con el fin de obtener una comprensión específica y enfocada de la percepción del bienestar subjetivo y analizar la influencia de la experiencia migratoria en el desarrollo personal, el conjunto de capacidades y la satisfacción con la vida de las mujeres migrantes.

\section{REFERENCIAS BIBLIOGRÁFICAS}

Allardt, E. (1993). Having, Loving, Being: An Alternative to the Swedish Model of Welfare Research. En: M. Nussbaum \& A. Sen (Eds.), The Quality of Life (pp.88-94). https://doi. org/10.1093/0198287976.003.0008

Amit, K. (2010). Determinants of Life Satisfaction Among Immigrants from Western Countries and from the FSU in Israel. Social Indicators Research, 96(3), 515534. https://doi.org/10.1007/s11205o09-9490-1

Arneson, R. J. (1999). Human Flourishing Versus Desire Satisfaction. Social Philosophy and Policy, 16(1), 113-142. https:// doi.org/10.1017/So265052500002272

Bak-Klimek, A., Karatzias, T., Elliott, L., \& Maclean, R. (2015). The Determinants of Well-Being Among International Economic Immigrants: A Systematic Literature Review and Meta-Analysis. Applied Research in Quality of Life, 10(1), 161188. https://doi.org/10.1007/s11482013-9297-8

Bartram, D. (2013). Happiness and 'economic migration': A comparison of Eastern European migrants and stayers. Migration Studies, 1(2), 156-175.

Baykara-Krumme, H., \& Platt, L. (2016). Life satisfaction of migrants, stayers and returnees: reaping the fruits of migration in old age? Ageing \& Society. Recupera- 
do de https://www.cambridge.org/core/ journals/ageing-and-society

Berry, J. W. (1997). Immigration, Acculturation, and Adaptation. Applied Psychology, 46(1), 5-34. https://doi. org/10.1111/j.1464-0597.1997.tbo1087.x

Csikszentmihalyi, M. (2008). Flow: The Psychology of Optimal Experience (1 edition). New York: Harper Perennial Modern Classics.

Cummins, R. A. (2010). Subjective Wellbeing, Homeostatically Protected Mood and Depression: A Synthesis. Journal of Happiness Studies, 11(1), 1-17. https:// doi.org/10.1007/s10902-009-9167-0

D’Isanto, F., Fouskas, P., \& Verde, M. (2016). Determinants of Well-being Among Legal and Illegal Immigrants: Evidence from South Italy. Social Indicators Research, 126(3), 1109-1141. https://doi. org/10.1007/s11205-015-0924-7

Diener, E., \& Suh, E. (1997). Measuring Quality of Life: Economic, Social, and Subjective Indicators. Social Indicators Research, 4O(1/2), 189-216.

Diener, E., Emmons, R., Larsen, R. J., \& Griffin, S. (1985). The Satisfaction With Life Scale. Journal of Personality Assessment, 49(1), 71-75. https://doi. org/10.1207/s15327752jpa4901_13

Diener, E., Suh, E. M., Lucas, R. E., \& Smith, H. L. (1999). Subjective well-being: Three decades of progress. Psychological Bulletin, 125(2), 276-302. https:// doi.org/10.1037/0033-2909.125.2.276

ESS. (s. f.). European Social Survey - Wellbeing matters. Recuperado 29 de mayo de 2017, de European Social Survey website: http://esswellbeingmatters.org/
Eurofound. (2003). Encuesta europea sobre calidad de vida (EQLS). Recuperado 15 de octubre de 2016, de http://www.eurofound.europa.eu/es/surveys/european-quality-of-life-surveys

Feldman, F. (2004). Pleasure and the Good Life: Concerning the Nature, Varieties and Plausibility of Hedonism. Clarendon Press.

fesEasterlin, R. A. (1974). Does Economic Growth Improve the Human Lot? Some Empirical Evidence. En Nations and Households in Economic Growth (pp. 89-125). https://doi.org/10.1016/B9780-12-205050-3.50008-7

Festinger, L. (1954). A Theory of Social Comparison Processes. Human Relations, $7(2), \quad$ 117-140. https://doi. org/10.1177/001872675400700202

Fitoussi, J. P., Sen, A., \& Stiglitz, J. E. (2009). Report by the Commission on the measurement of economic performance and social progress (p.292). Recuperado de https://www.mysciencework.com/publication/show/502ed1c8e1eobc2855b757c509cce4c9

Fortin, N. M., Helliwell, J. F., \& Wang, S. (2015). How Does Subjective Well-being Vary around the World by Gender and Age? En World Happiness Report Series: Cap. 3. World Happiness Report 2015 Launched (Sustainable Development Solutions Network). John Helliwell, Richard Layard, Jeffrey Sachs.

Gallup, Inc. (s. f.). Gallup Global Wellbeing. Recuperado 24 de abril de 2017, de Gallup.com website: http://www.gallup. com/poll/126965/Gallup-Global-Wellbeing.aspx 
Goodwin, R., Cook, O., \& Yung, Y. (2001). Loneliness and life satisfaction among three cultural groups. Personal Relationships, 8(2), 225-230. https://doi. org/10.1111/j.1475-6811.2001.tbooo37.x

Hajdu, G., \& Hajdu, T. (2016). The Impact of Culture on Well-Being: Evidence from a Natural Experiment. Journal of Happiness Studies, 17(3), 1089-1110. https:// doi.org/10.1007/s10902-015-9633-9

Haybron, D. M. (2008). Happiness, the Self and Human Flourishing. Utilitas, 2O(1), 21-49. https://doi.org/10.1017/ So953820807002889

Hendriks, M. (2015). The happiness of international migrants: A review of research findings. Migration Studies, 3(3), 343369. https://doi.org/10.1093/migration/mnuo53

Hendriks, M., \& Bartram, D. (2016). Macro-conditions and immigrants' happiness: Is moving to a wealthy country all that matters? Social Science Research, 56(Supplement C), 90-107. https://doi. org/10.1016/j.ssresearch.2015.11.006

Hendriks, M., \& Bartram, D. (2018). Bringing Happiness Into the Study of Migration and Its Consequences: What, Why, and How? Journal of Immigrant \& Refugee Studies, 1-20. https://doi.org/10.1 080/15562948.2018.1458169

Huppert, F., Clark, A., Stutzer, A., \& Vittersø, J. (2009). ESS 3 - Personal and Social Wellbeing Module for the European Social Survey (N.o NC8 Paper 3b). London, Centre for Comparative Social Surveys, City University London: European Social Survey.

Ivlevs, A. (2015). Happy Moves? Assessing the Link between Life Satisfaction and
Emigration Intentions: Life Satisfaction and the Emigration Decision. Kyklos, 68(3), 335-356. https://doi.org/10.1111/ kykl.12086

Kahneman, D. (2012). Pensieri lenti e veloci (L. Serra, Trad.). Edizioni Mondadori.

Kahneman, D., \& Krueger, A. B. (2006). Developments in the Measurement of Subjective Well-Being. Journal of Economic Perspectives, 20(1), 3-24. https://doi. org/10.1257/089533006776526030

Keller, S. (2004). Welfare and the Achievement of Goals. Philosophical Studies, 121(1), 27-41. https://doi.org/10.1023/ B:PHIL.0000040377.50002.88

Keller, S. (2009). Welfare as Success. Noûs, 43(4), 656-683.

Kraut, R. (1979). Two Conceptions of Happiness. Philosophical Review, 88(2), 167197.

Lukas, M. (2010). Desire Satisfactionism and the Problem of Irrelevant Desires. Journal of Ethics \& Social Philosophy, 1.

Lyubomirsky, S., Sheldon, K. M., \& Schkade, D. (2005). Pursuing Happiness: The Architecture of Sustainable Change. Review of General Psychology, 9(2), 111-131. https://doi.org/10.1037/10892680.9.2.111

Max-Neef, M. A., Elizalde, A., \& Hopenhayn, M. (1994). Desarrollo a escala humana: conceptos, aplicaciones y algunas reflexiones. Icaria Editorial.

Murphy, M. C. (1999). The Simple Desire-Fulfillment Theory. Noûs, 33(2), 247-272. https://doi.org/10.1111/o0294624.00153

Nesterko, Y., Braehler, E., Grande, G., \& Glaesmer, H. (2013). Life satisfaction 
and health-related quality of life in immigrants and native-born Germans: the role of immigration-related factors. Quality of Life Research, 22, 1005-1013. Nikolova, M., \& Graham, C. (2015). In transit: The well-being of migrants from transition and post-transition countries. Journal of Economic Behavior \& Organization, 112, 164-186. https://doi. org/10.1016/j.jebo.2015.02.003

Nussbaum, M. C. (2001). Women and Human Development: The Capabilities Approach. Cambridge University Press.

Nussbaum, M. C. (2011). The central capabilities. En M. C. Nussbaum, Creating Capabilities. The Human Development Approach (pp.17-45). Cambridge: Harvard University Press.

Nussbaum, M., \& Sen, A. (1993). The Quality of Life (World Institute for Development Economics Research). Oxford University Press.

OECD. (2011). Compendium of OECD well-being indicators (pp.1-37). Recuperado de OECD website: http://www. oecd.org/general/compendiumofoecdwell-beingindicators.htm

OECD. (2015). How's Life? 2015: Measuring Well-being (OECD Publishing). Paris: OECD.

OECD. (2017). How's Life? 2017: Measuring Well-being (OECD Publishing). Recuperado de http://www.oecd.org/statistics/how-s-life-23089679.htm

OIM. (2010). World migration report 2010 the future of migration: building capacities for change. Ginebra: Organización Internacional para las Migraciones. (ISSN 1561-5502).

OIM. (2013). Informe sobre las migraciones en el mundo 2013. El Bienestar de los Migrantes y el Desarrollo. Ginebra: Organización Internacional para las Migraciones. (ISSN 1561-5502).

Olgiati, A., Calvo, R., \& Berkman, L. (2013). Are Migrants Going Up a Blind Alley? Economic Migration and Life Satisfaction around the World: Cross-National Evidence from Europe, North America and Australia. Social Indicators Research, 114(2), 383-404. https://doi. org/10.1007/s11205-012-0151-4

Raibley, J. R. (2012). Happiness is not Well-Being. Journal of Happiness Studies, 13(6), 1105-1129. https://doi. org/10.1007/s10902-011-9309-Z

Richardson, A. (1974). British immigrants and Australia: a psychosocial inquiry. Canberra: Australian National University Press [distributed in the U.S. by International Scholarly Book Services, Portland, Oregon].

Robeyns, I. (2003). Sen's Capability Approach and Gender Inequality: Selecting Relevant Capabilities. Feminist Economics, 9(2-3), 61-92. https://doi. org/10.1080/1354570022000078024

Robeyns, I. (2005). The Capability Approach: a theoretical survey. Journal of Human Development, 6(1), 93-117. https://doi. org/10.1080/146498805200034266

Safi, M. (2010). Immigrants' Life Satisfaction in Europe: Between Assimilation and Discrimination. European Sociological Review, 26(2), 159-176. https://doi. org/10.1093/esr/jcpo13

Scanlon, T. (1998). What We Owe to Each Other. Belknap Press of Harvard University Press. 
Sen, A. (1985). Well-Being, Agency and Freedom: The Dewey Lectures 1984. The Journal of Philosophy, 82(4), 169-221. https://doi.org/10.2307/2026184

Sen, A. (1987). The Standard of Living. En G. Hawthorn (Ed.), The Standard of Living. Cambridge: Cambridge University Press.

Sen, A. (1999). Commodities and Capabilities. OUP India.

Sumner, L. W. (1996). Welfare, Happiness, and Ethics. New York: Oxford University Press.

Veenhoven, R. (2000). The Four Qualities of Life. Journal of Happiness Studies, 1(1), 1-39. https://doi. org/10.1023/A:1010072010360

Verkuyten, M. (2008). Life Satisfaction Among Ethnic Minorities: The Role of Discrimination and Group Identification. Social Indicators Research, 89(3), 391-404. https://doi.org/10.1007/
S11205-008-9239-2

Voicu, B., \& Vasile, M. (2014). Do 'cultures of life satisfaction' travel? Current Sociology, 62(1), 81-99. https://doi. org/10.1177/o011392113516651

Vroome, T. de, \& Hooghe, M. (2014). Life Satisfaction among Ethnic Minorities in the Netherlands: Immigration Experience or Adverse Living Conditions? Journal of Happiness Studies, 15(6), 1389-1406. https://doi.org/10.1007/s10902-0139483-2

\section{PARA CITAR ESTE ARTículo}

Panzeri, R. (2019). Bienestar subjetivos de migrantes: la ausencia de la dimensión de género. Collectivus, Revista de Ciencias Sociales, 6(2), 33-58

DOI: http://dx.doi.org/10.15648/Coll.2.2019.3

Recibido: 10/11/2018 Aprobado: 12/02/2019 


\begin{tabular}{|c|c|c|c|}
\hline 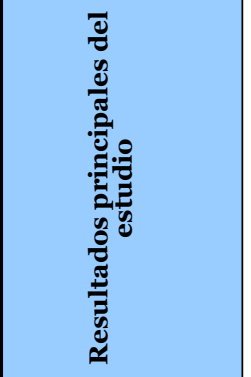 & 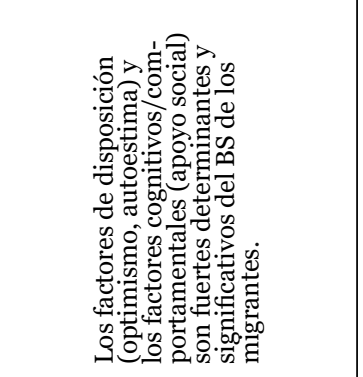 & 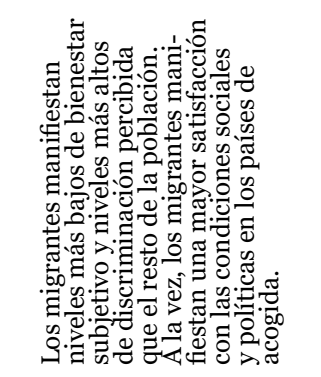 & 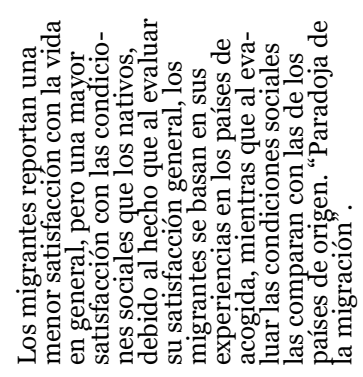 \\
\hline 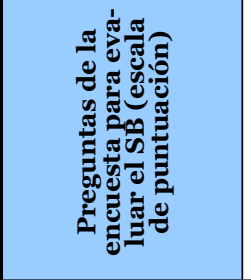 & $\approx$ & 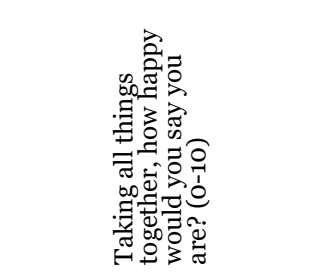 & 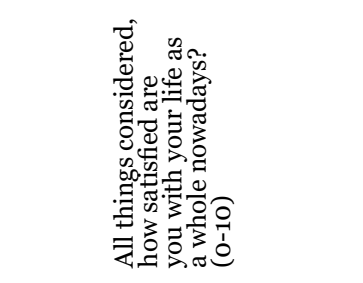 \\
\hline 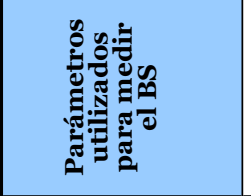 & 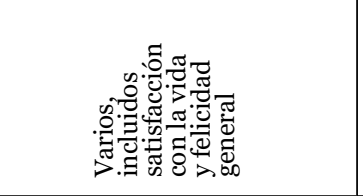 & 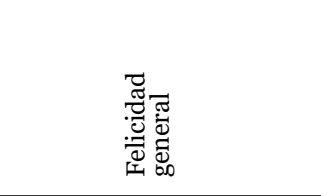 & 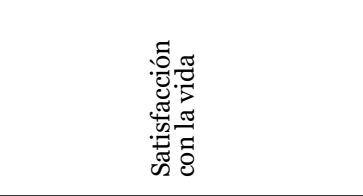 \\
\hline 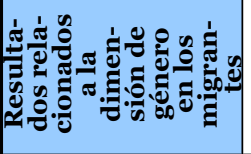 & 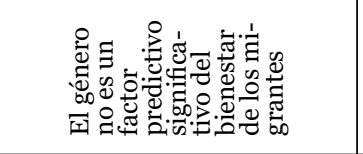 & 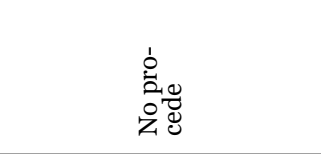 & 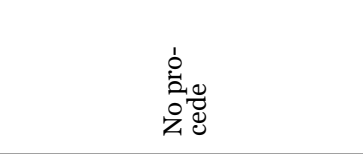 \\
\hline 今. & 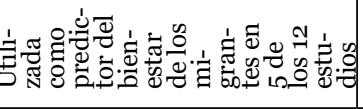 & $\stackrel{8}{z}$ & $\stackrel{\circ}{Z}$ \\
\hline 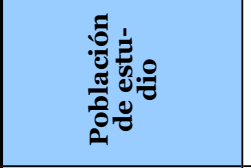 & 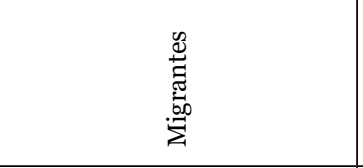 & 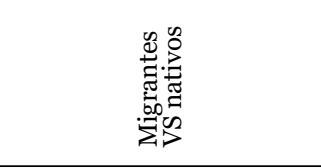 & 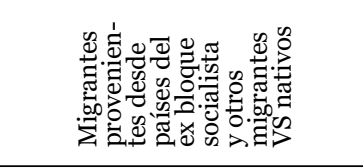 \\
\hline 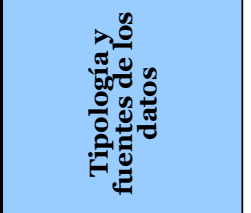 & 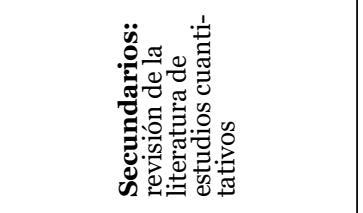 & 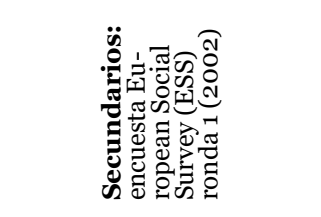 & 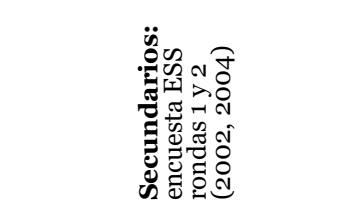 \\
\hline 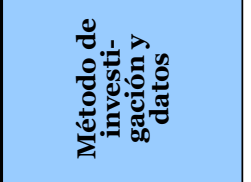 & 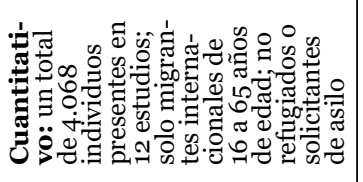 & 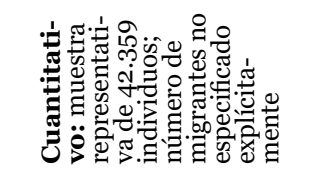 & 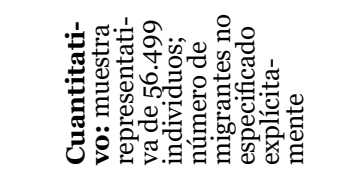 \\
\hline 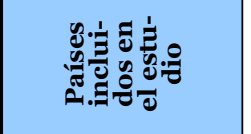 & 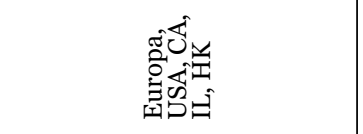 & 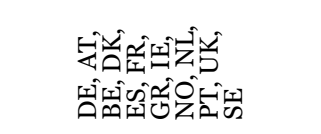 & 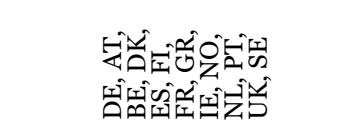 \\
\hline 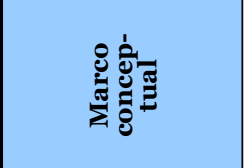 & 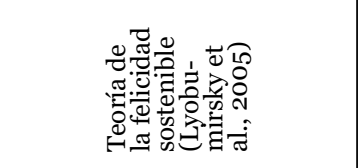 & 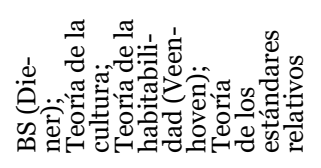 & 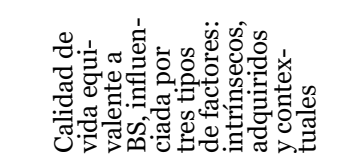 \\
\hline o!pmst & 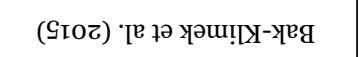 & (ভоoz) nosәрең[еg & (Looz) nәsәғетгеg \\
\hline
\end{tabular}




\begin{tabular}{|c|c|c|}
\hline 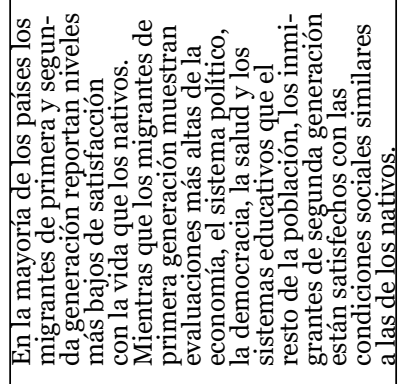 & 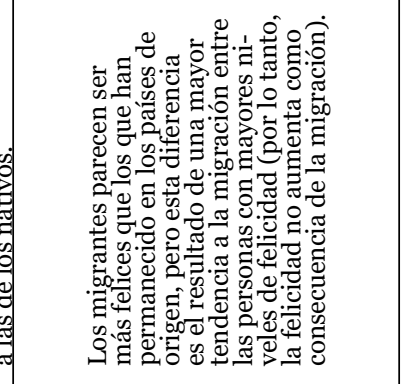 & 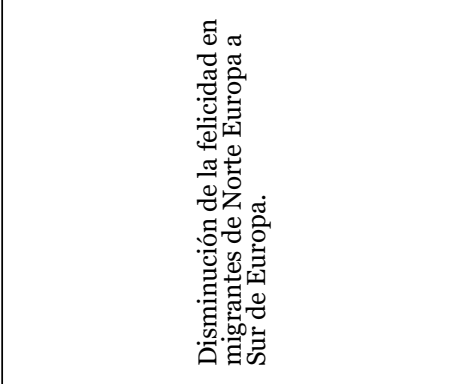 \\
\hline 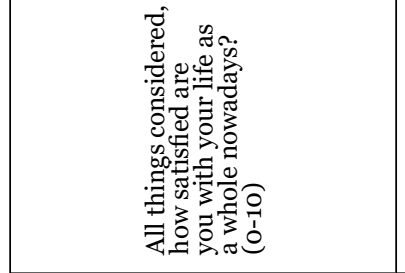 & 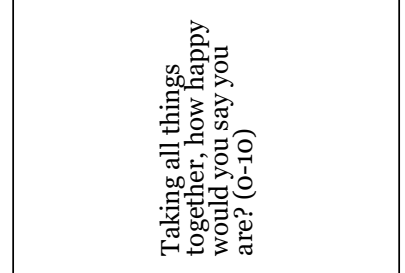 & 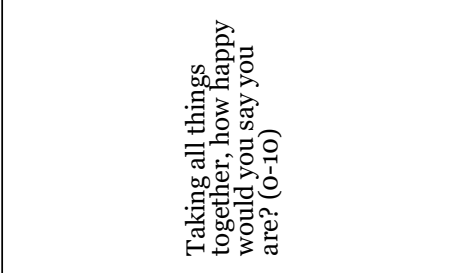 \\
\hline 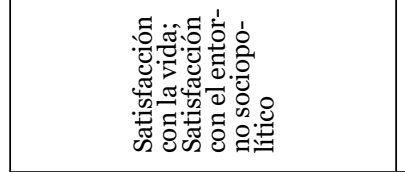 & 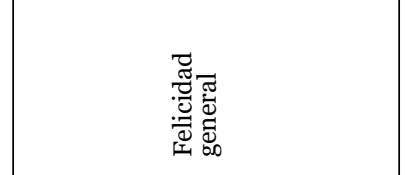 & 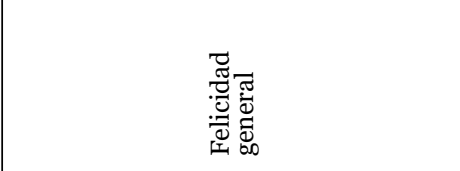 \\
\hline 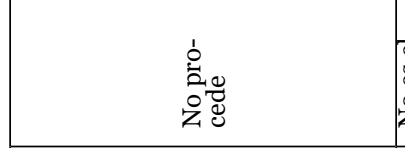 & 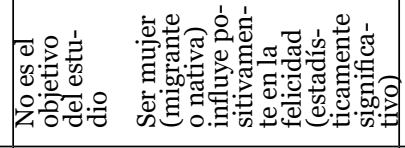 & 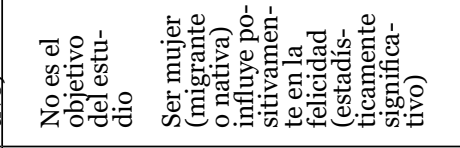 \\
\hline そ & 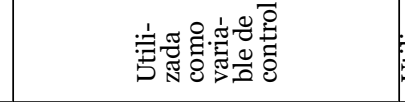 & 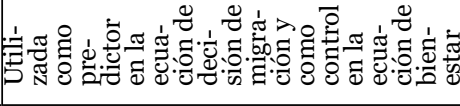 \\
\hline 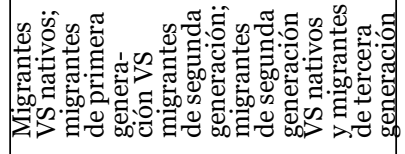 & 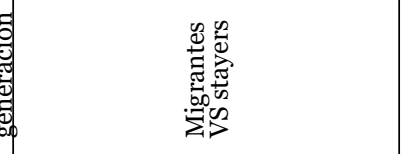 & 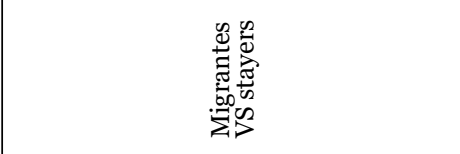 \\
\hline 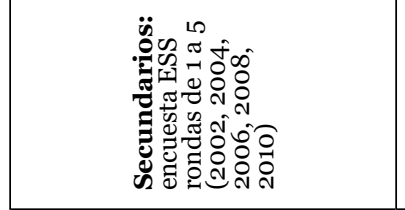 & 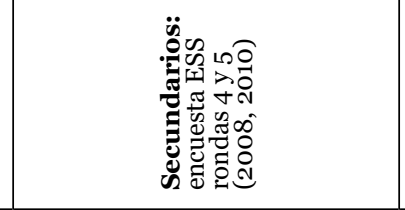 & 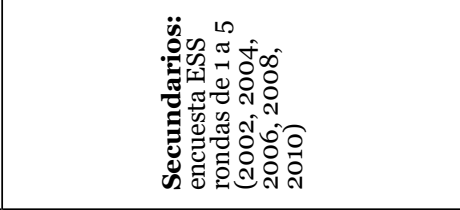 \\
\hline 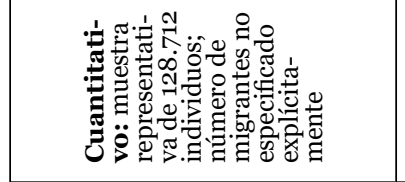 & 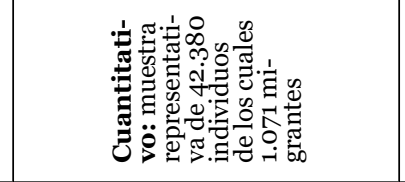 & 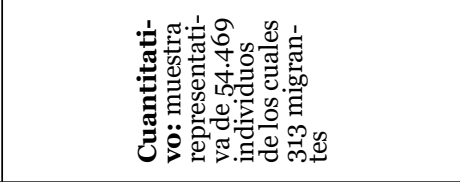 \\
\hline 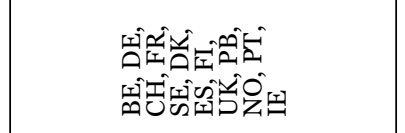 & 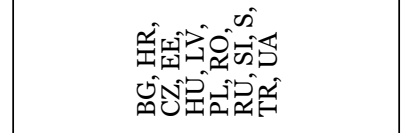 & 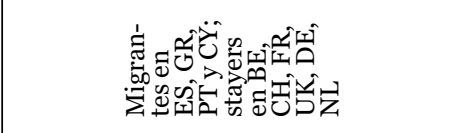 \\
\hline 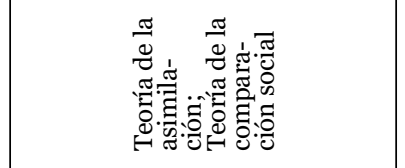 & 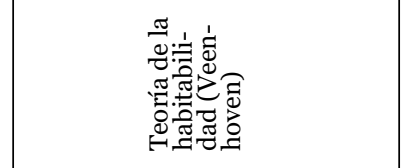 & 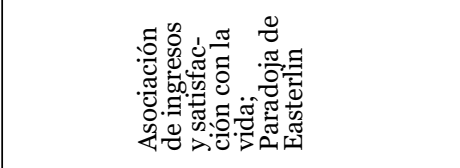 \\
\hline (†тоz) nosәреұ[eg & (Eเоz) ше.п.кеg & (ভั0z) uе.n.reg \\
\hline
\end{tabular}




\begin{tabular}{|c|c|c|}
\hline 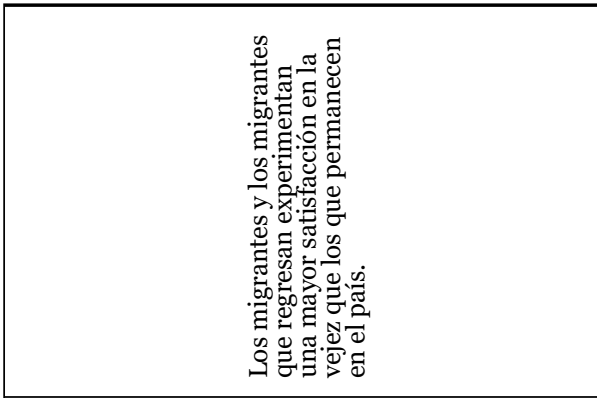 & 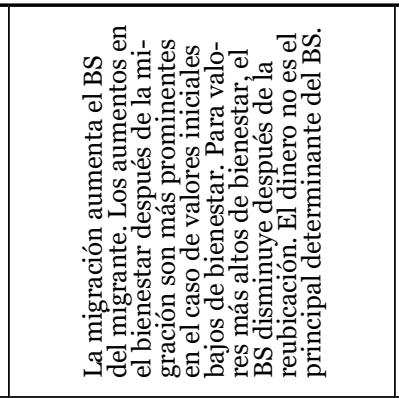 & 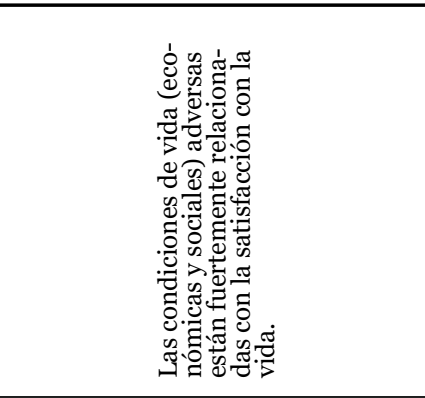 \\
\hline 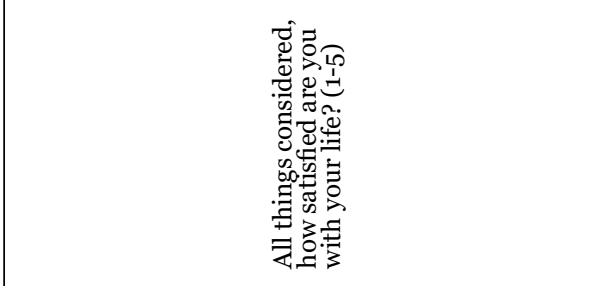 & 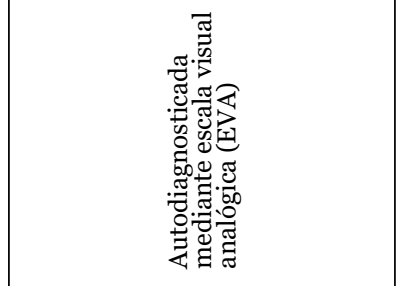 & 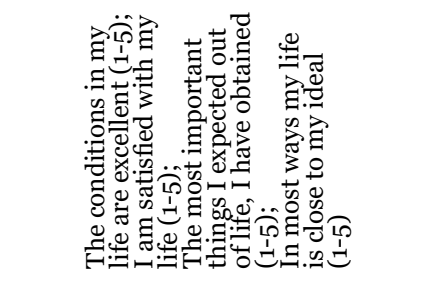 \\
\hline 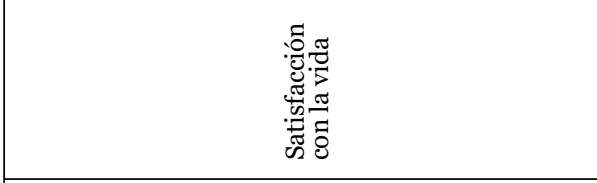 & 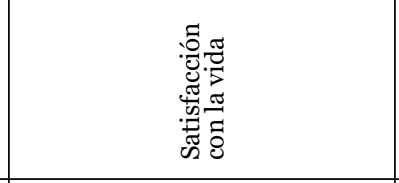 & 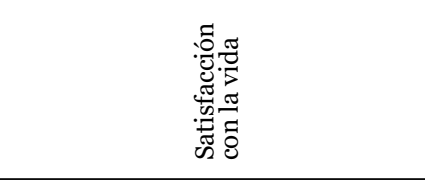 \\
\hline 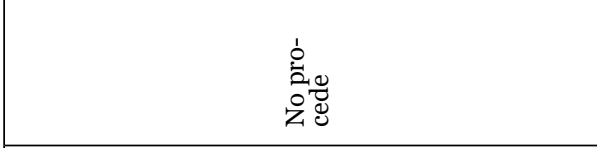 & 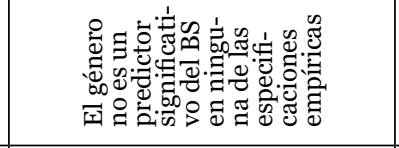 & 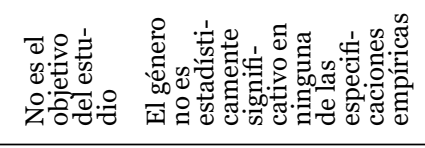 \\
\hline 要 & 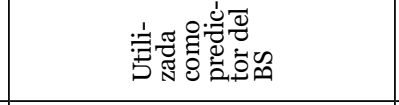 & 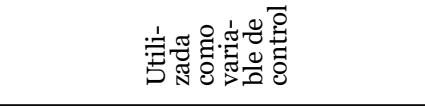 \\
\hline 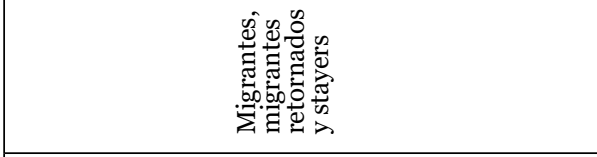 & 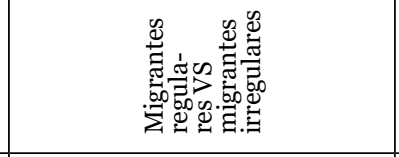 & 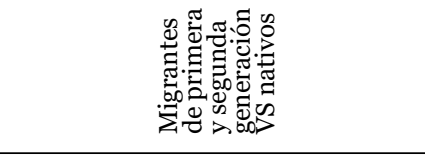 \\
\hline 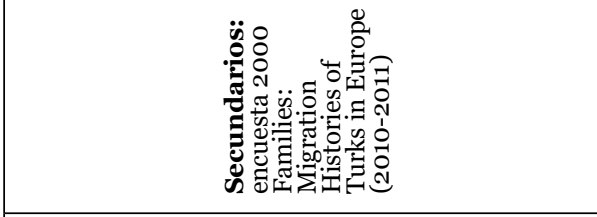 & 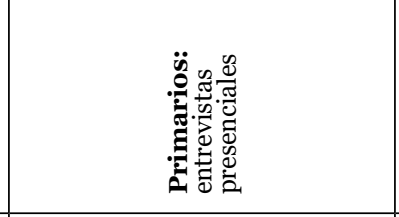 & 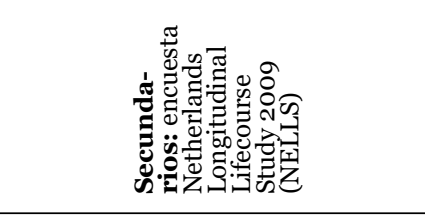 \\
\hline 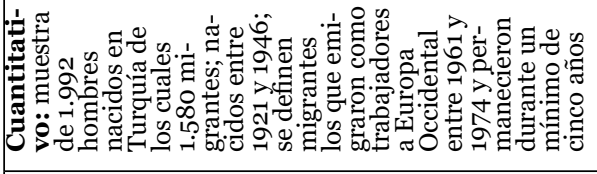 & 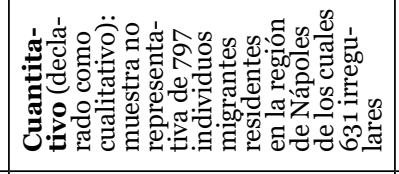 & 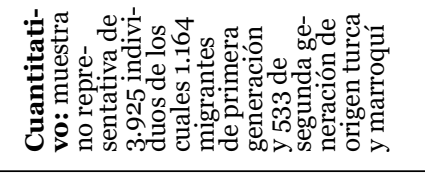 \\
\hline 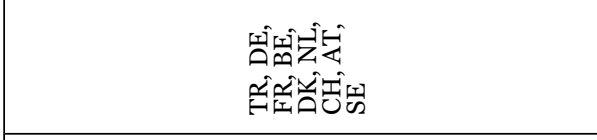 & $\Xi$ & $\vec{z}$ \\
\hline 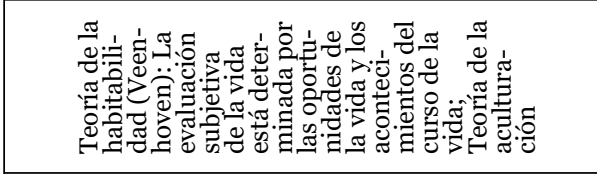 & 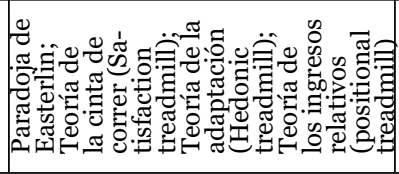 & 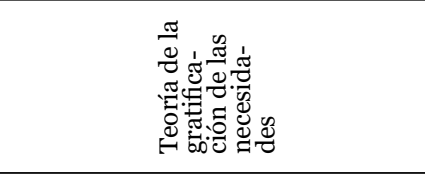 \\
\hline 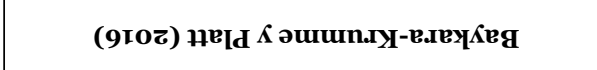 & 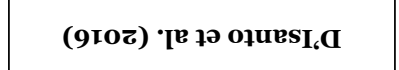 & 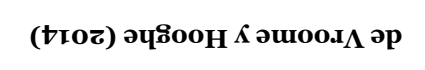 \\
\hline
\end{tabular}




\begin{tabular}{|c|c|c|}
\hline 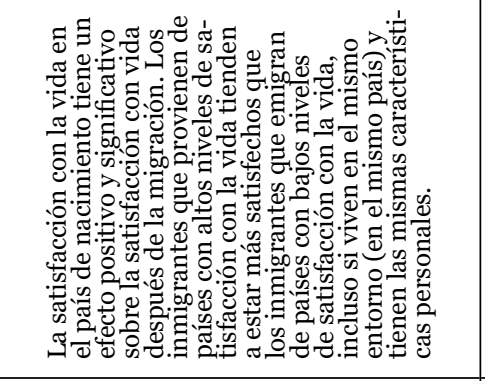 & 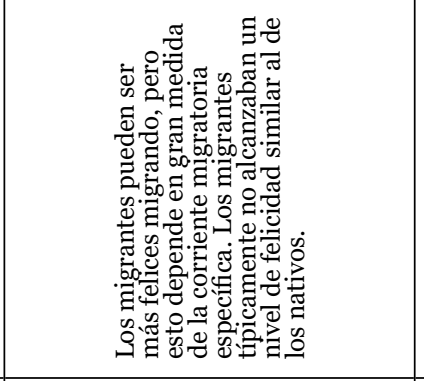 & 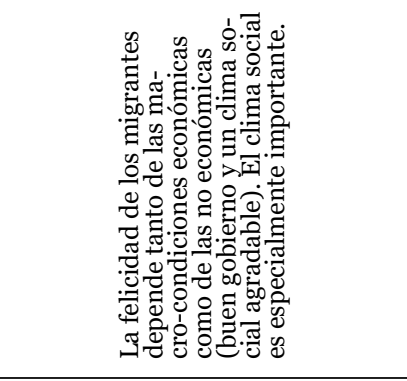 \\
\hline 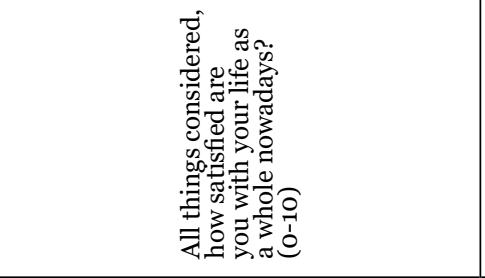 & $=$ & 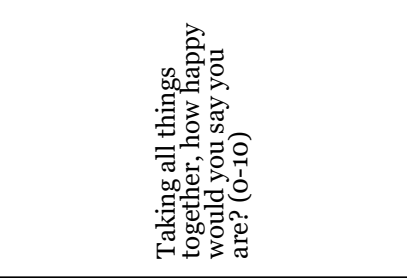 \\
\hline 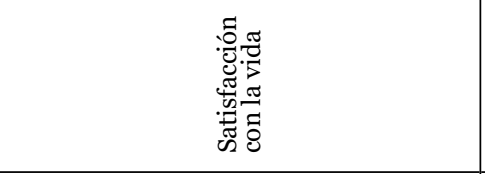 & 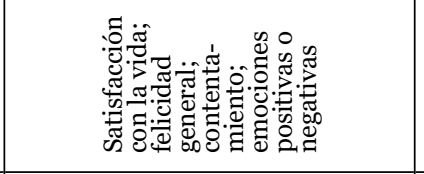 & 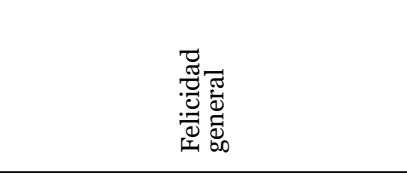 \\
\hline 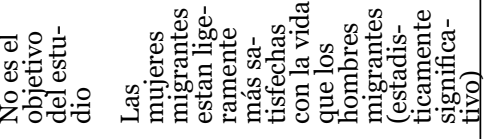 & 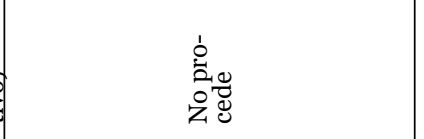 & 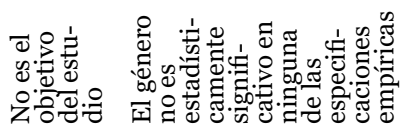 \\
\hline 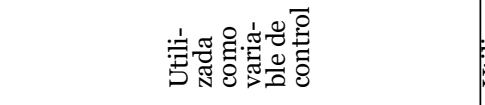 & 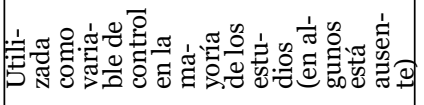 & 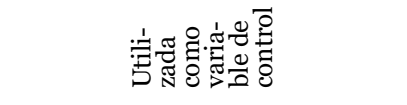 \\
\hline 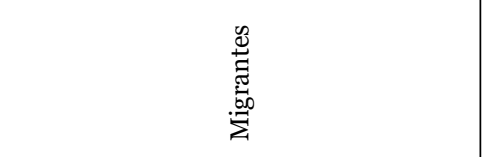 & 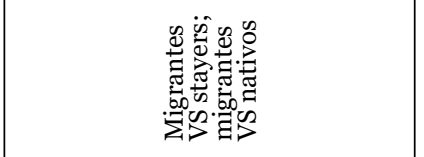 & 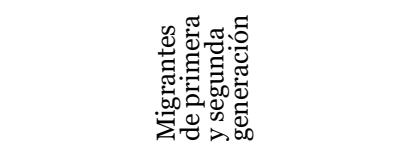 \\
\hline 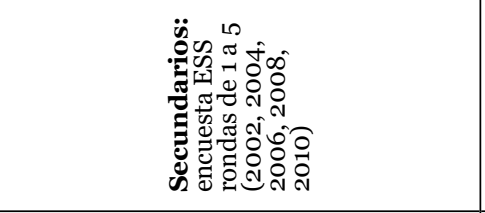 & 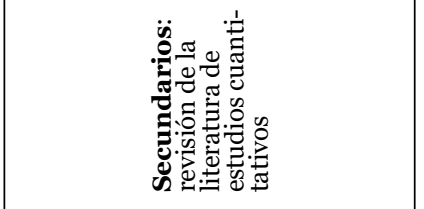 & 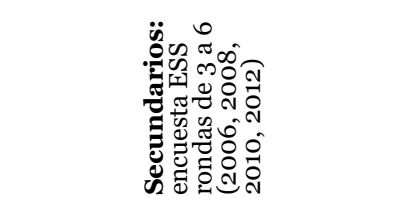 \\
\hline 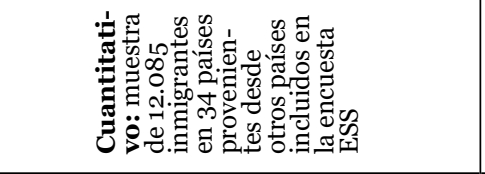 & 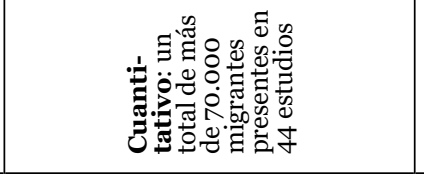 & 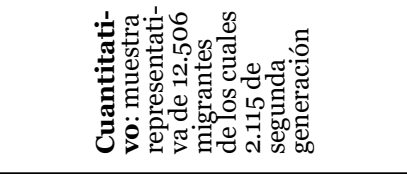 \\
\hline 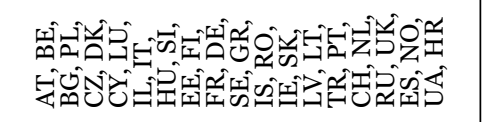 & 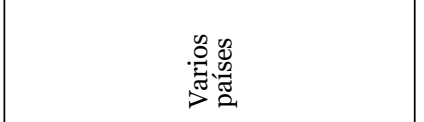 & 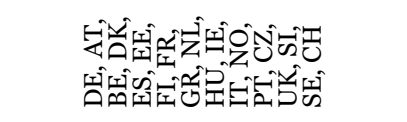 \\
\hline 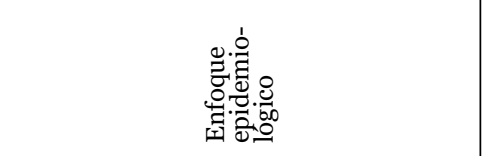 & 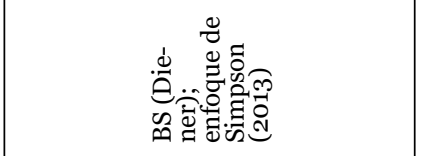 & 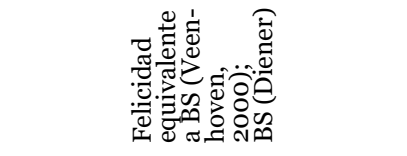 \\
\hline 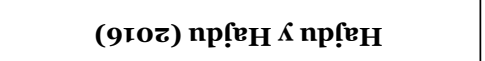 & 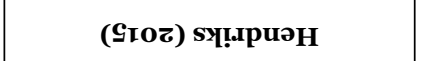 & (9roz) ur.n.reg $A$ sy!.,puән \\
\hline
\end{tabular}




\begin{tabular}{|c|c|c|}
\hline 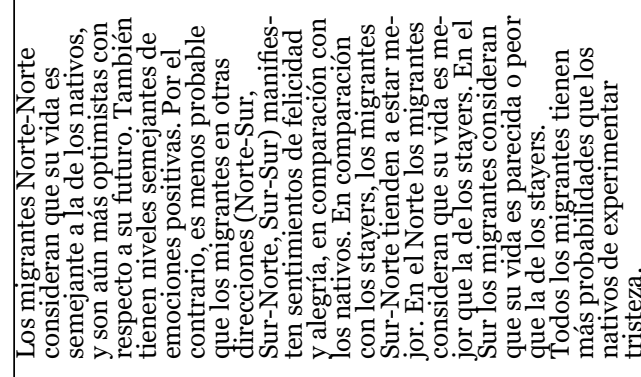 & 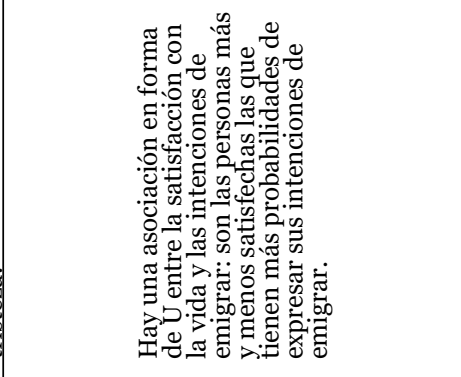 & 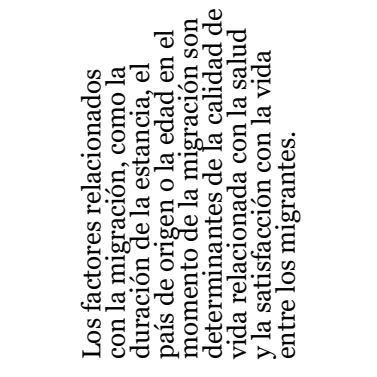 \\
\hline 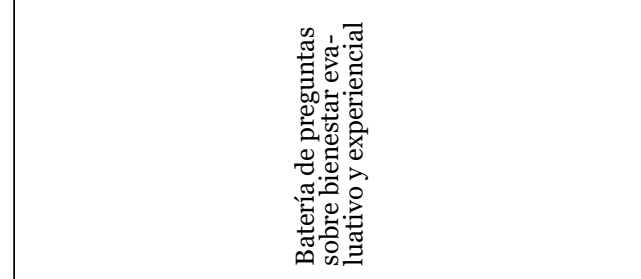 & 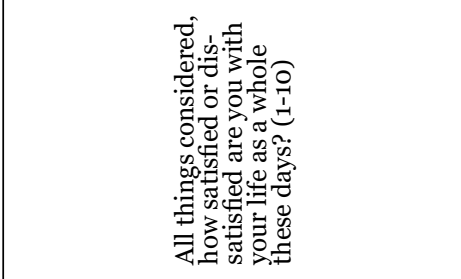 & 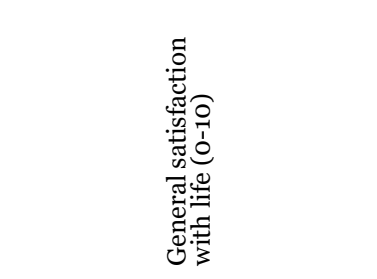 \\
\hline 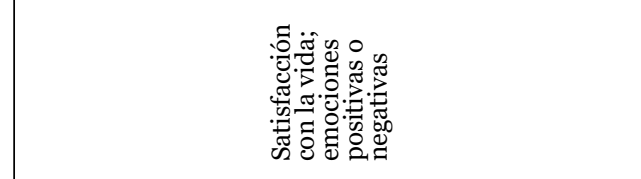 & 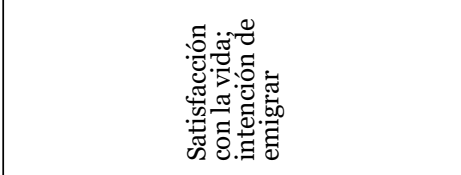 & 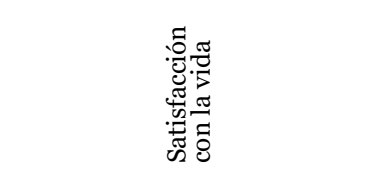 \\
\hline 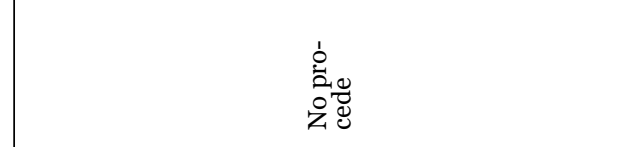 & 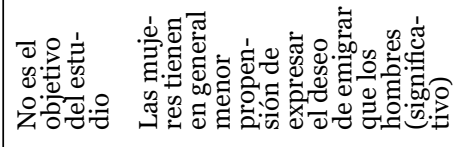 & 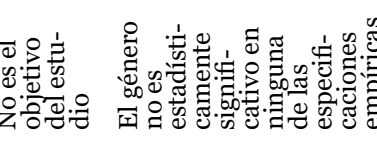 \\
\hline そ & 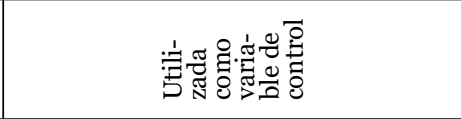 & 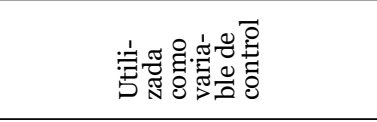 \\
\hline 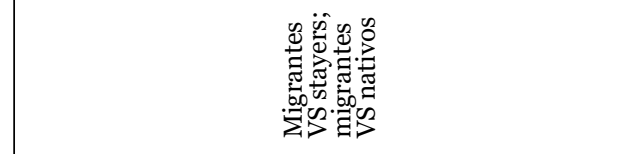 & 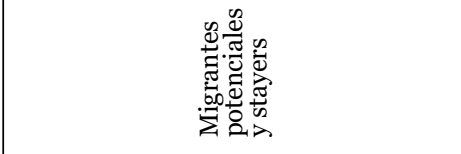 & 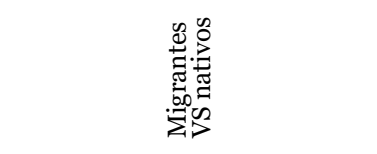 \\
\hline 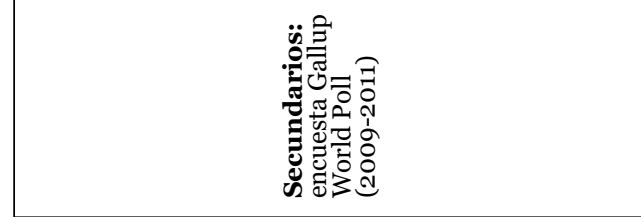 & 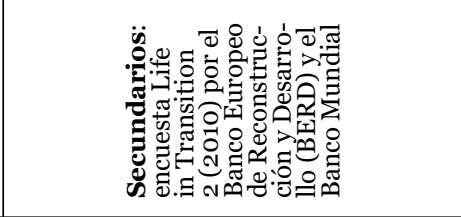 & 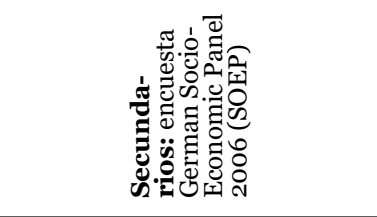 \\
\hline 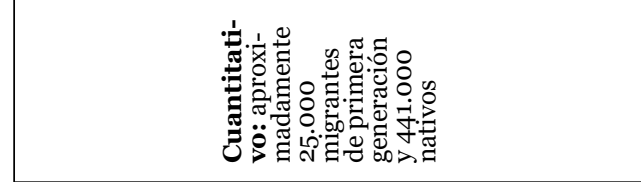 & 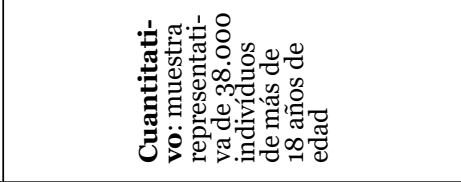 & 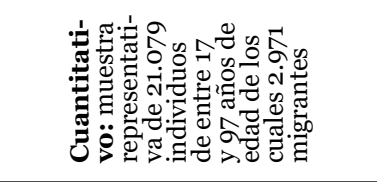 \\
\hline 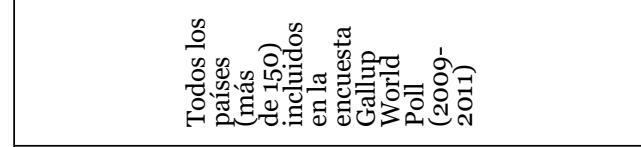 & 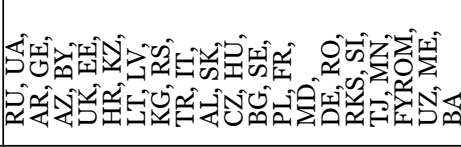 & 됨 \\
\hline 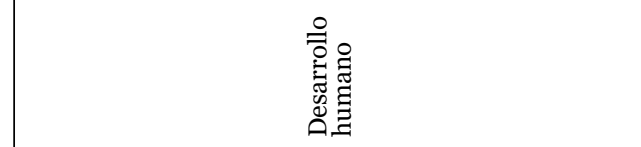 & 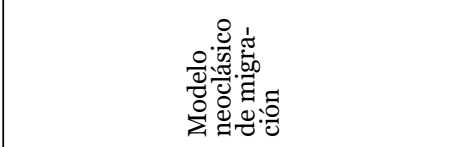 & 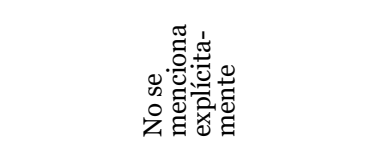 \\
\hline (Eเоб) ИIO әш.лојиI & 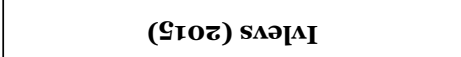 & (EI0б) ' ‘ \\
\hline
\end{tabular}




\begin{tabular}{|c|c|}
\hline 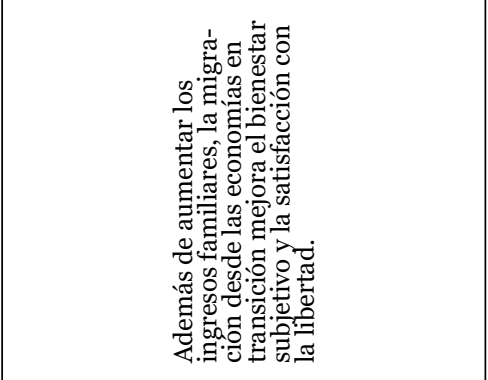 & 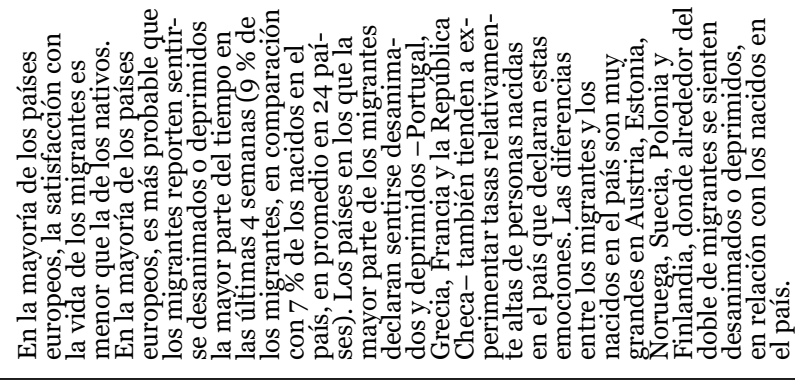 \\
\hline 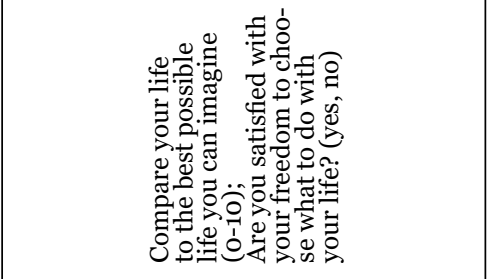 & 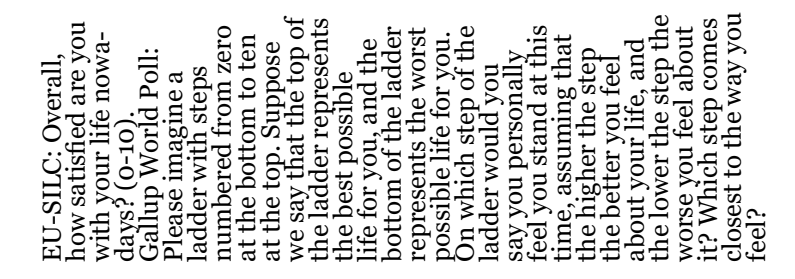 \\
\hline 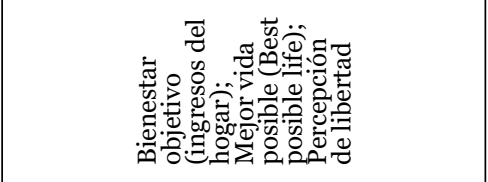 & 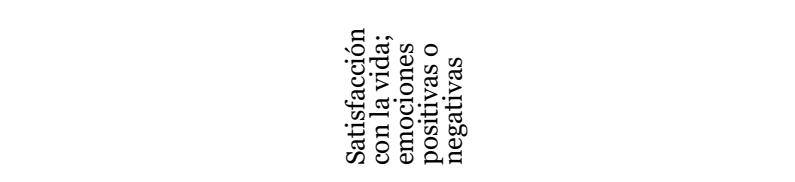 \\
\hline 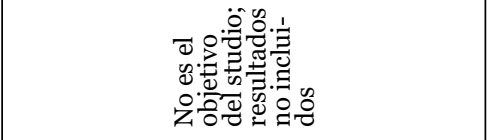 & 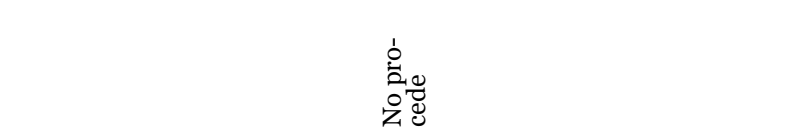 \\
\hline 击孚 & $\stackrel{\circ}{z}$ \\
\hline 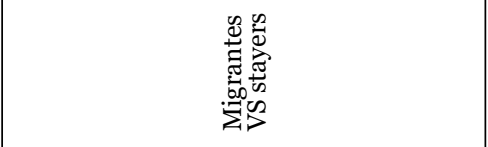 & 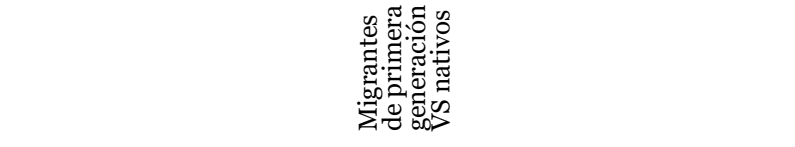 \\
\hline 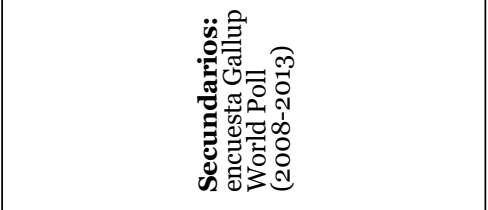 & 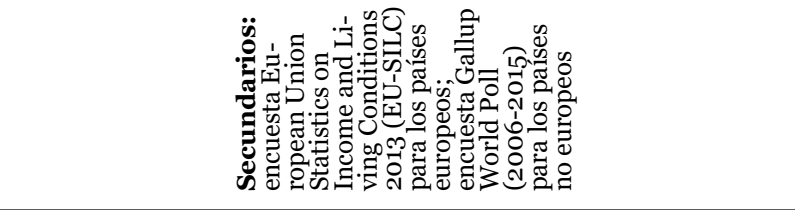 \\
\hline 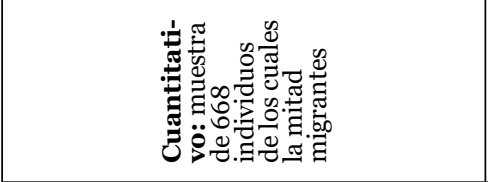 & 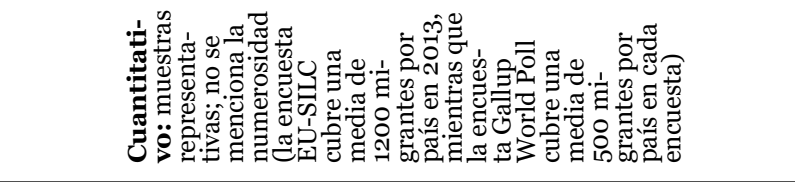 \\
\hline 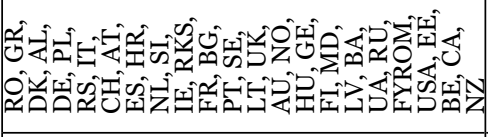 & 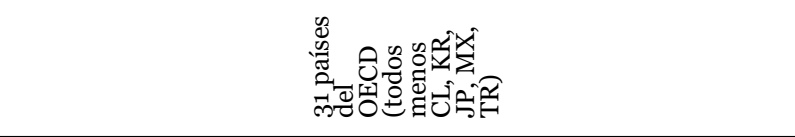 \\
\hline 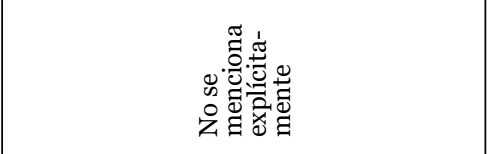 & 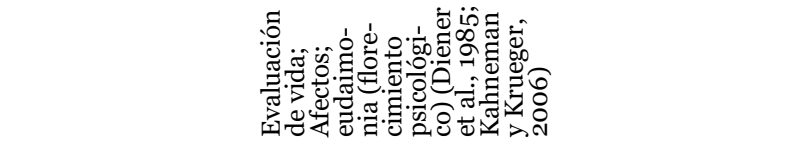 \\
\hline 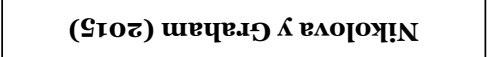 & (८нов) ваЈо \\
\hline
\end{tabular}




\begin{tabular}{|c|c|}
\hline 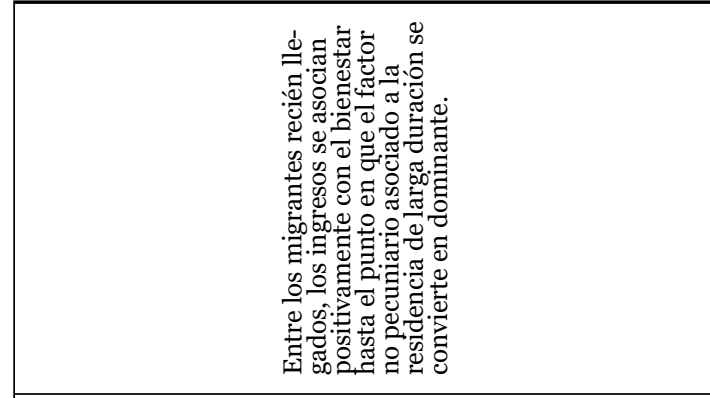 & 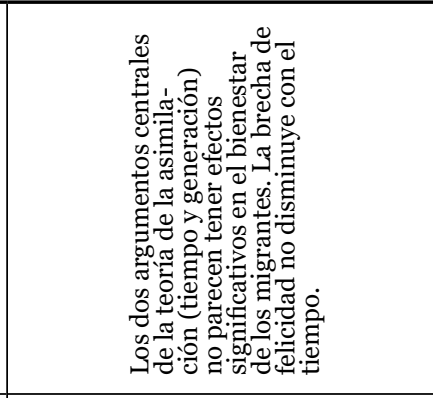 \\
\hline 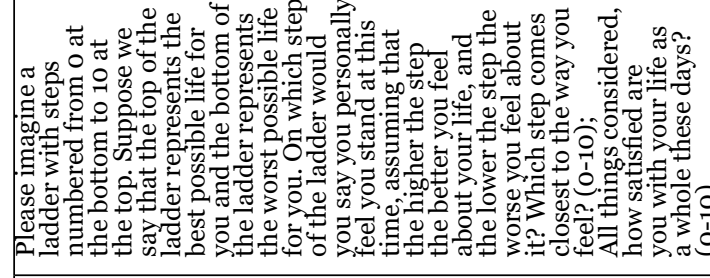 & 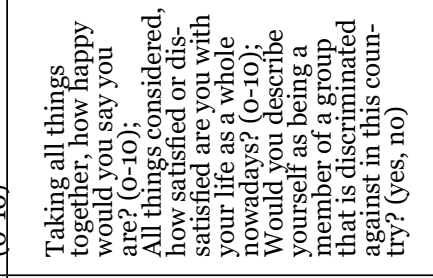 \\
\hline 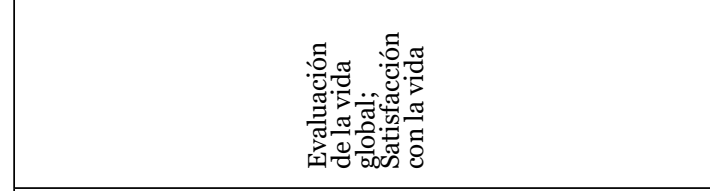 & 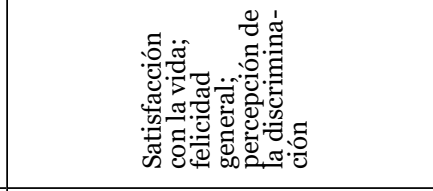 \\
\hline 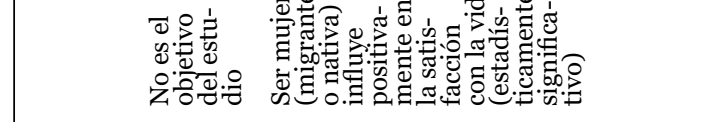 & 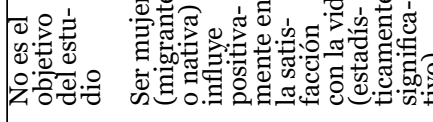 \\
\hline 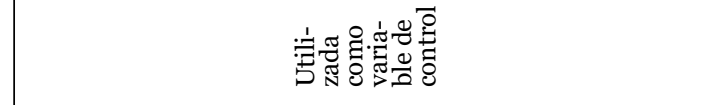 & \\
\hline 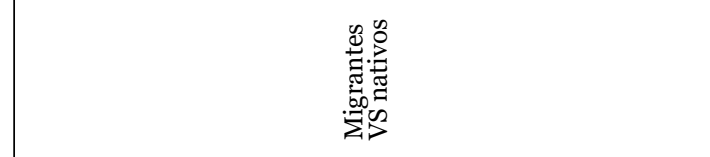 & 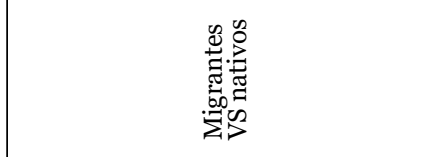 \\
\hline 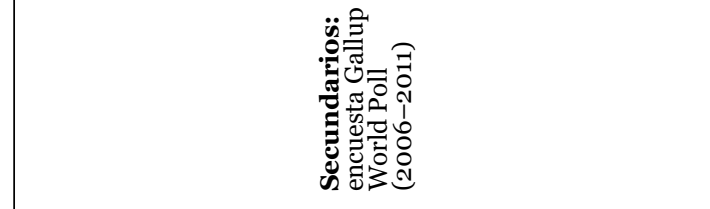 & 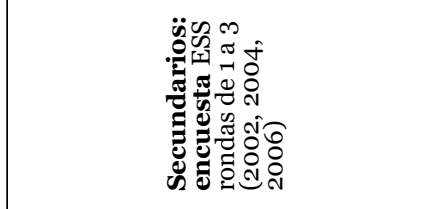 \\
\hline 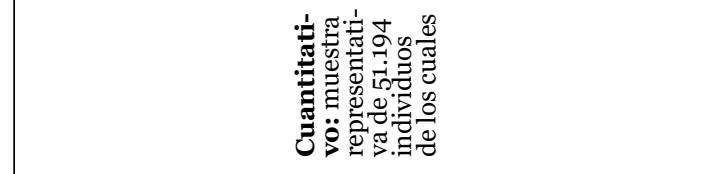 & 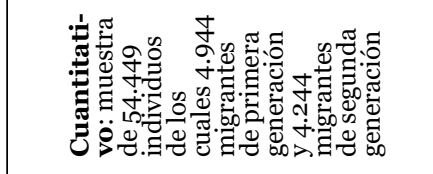 \\
\hline 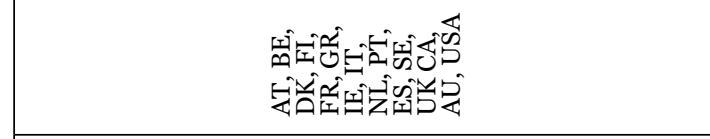 & 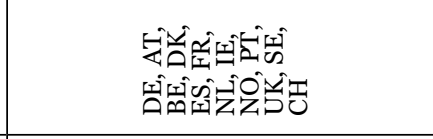 \\
\hline 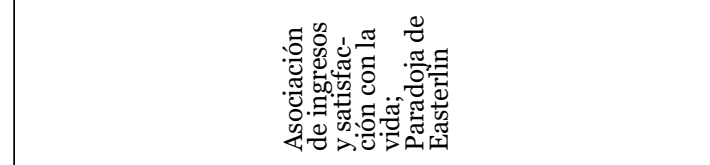 & 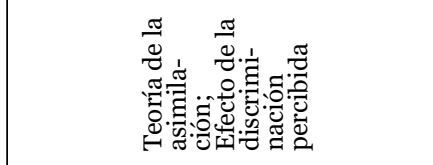 \\
\hline (Eเоz) & (Oroz) yes \\
\hline
\end{tabular}




\begin{tabular}{|c|c|}
\hline 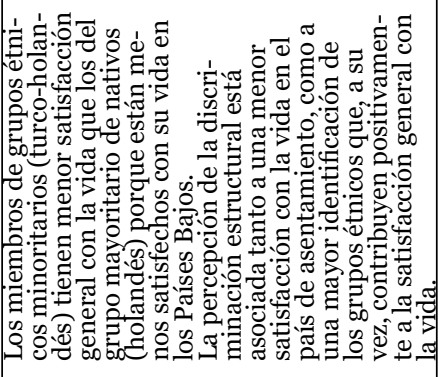 & 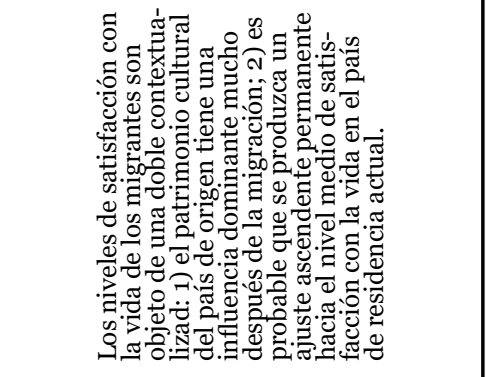 \\
\hline 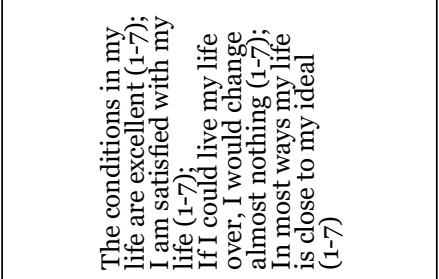 & 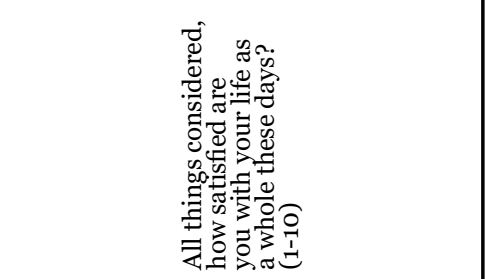 \\
\hline 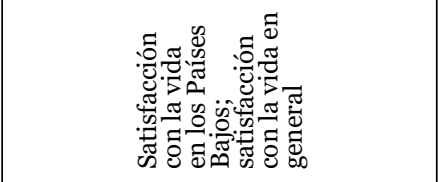 & 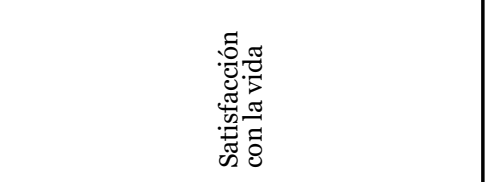 \\
\hline 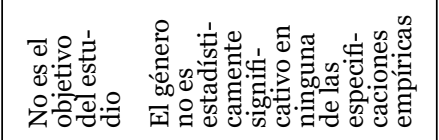 & 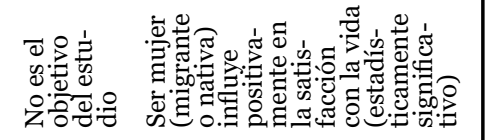 \\
\hline 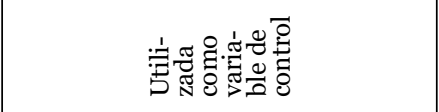 & 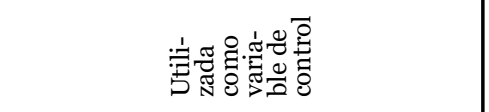 \\
\hline 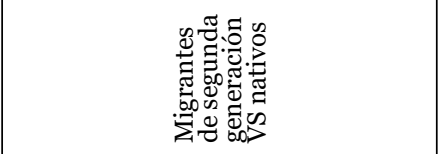 & 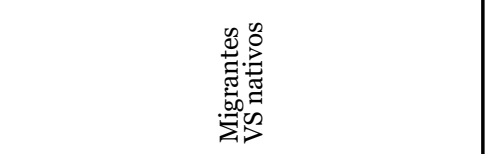 \\
\hline 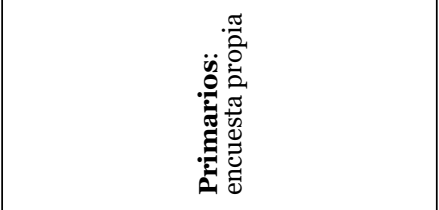 & 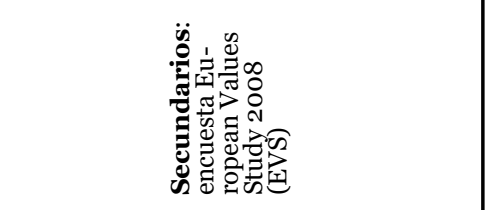 \\
\hline 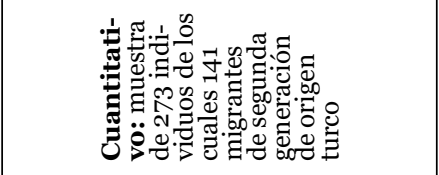 & 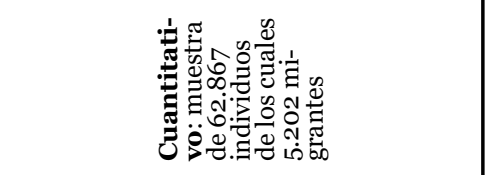 \\
\hline$\vec{z}$ & 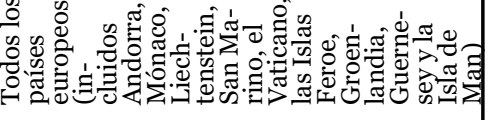 \\
\hline 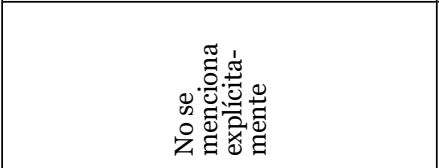 & 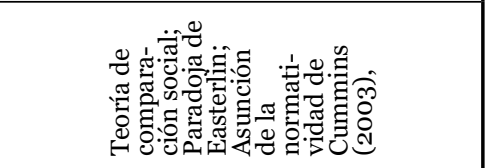 \\
\hline 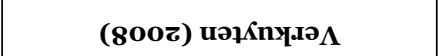 & 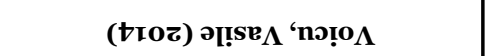 \\
\hline
\end{tabular}

